\title{
Modeling of micro-perforated panels in a complex vibro-acoustic environment using Patch Transfer Function approach
}

\author{
L. Maxit ${ }^{(1)}$, C. Yang ${ }^{(2)}$, L. Cheng ${ }^{(2)}$, J.-L. Guyader ${ }^{(1)}$
}

(1), Laboratoire Vibrations-Acoustique, INSA Lyon, 25 bis, av. Jean Capelle, 69621 Villeurbanne Cedex - France

(2), The Hong Kong Polytechnic University, Department of Mechanical Engineering, Kowloon, Hong Kong

Running title: MPP absorber in complex environment 


\begin{abstract}
The micro-perforated panel (MPP) with a backing cavity is a well known efficient device for noise absorption. This device has been thoroughly studied in the experimental conditions of an acoustic tube (Kundt tube), in which the MPP is excited by a normal incident plane wave in one dimension. In an industrial situation, the efficiency of MPP may be influenced by the vibro-acoustic behaviour of the surrounding systems as well as excitation. To deal with this problem, a vibroacoustic formulation based on the Patch Transfer Functions (PTF) approach is proposed to model the behaviour of micro-perforated structure in a complex vibro-acoustic environment. PTF is a substructuring approach which allows assembling different vibroacoustic subsystems through coupled surfaces. Upon casting micro-perforations and the flexibility of the MPP under transfer function framework, the proposed PTF formulation provides explicit representation of the coupling between subsystems and facilitates explanation of physical phenomenon. As an illustration example, application to a MPP with a backing cavity located in an infinite baffle is demonstrated. The proposed PTF formulation is finally validated through comparison with experimental measurements available in the literature.
\end{abstract}

PACS numbers: 43.50.Gf; 43.55.Ev 


\section{Introduction}

Since the pioneer work by Maa [1], micro-perforated panels (MPP) have been extensively used to design various sound absorption devices. The basic configuration is the one in which an air gap/cavity is placed at the back of the MPP, which creates a Helmholtz sound absorption effect, resulting in effective sound absorption. This basic configuration has been thoroughly studied both theoretically and experimentally using an acoustic tube (Kundt tube) in order to quantify its absorption coefficient. In such situation, the system has one single dimension and the MPP is excited by a normal incident plane wave. The prevailing motivation behind these efforts is the assumption that, the acoustic property of the MPP is assumed to locally reactive. Upon obtaining the surface acoustic impedance or the sound absorption coefficient of the MPP, it will be treated as conventional sound absorption materials.

Meanwhile, in the pursuit of a more efficient sound absorption, effort has also been made to use MPP in forming more complex system which deviates more or less from the basic configuration mentioned above. Such devices, taking various forms, can loosely be refereed as Micro-Perforated Structure (MPS). Double layer [2] or multiple layer absorbers [3] using MPPs are typical examples. The insertion of additional micro-perforated panel increases the acoustic resistance of the absorber and extends the effective absorption band toward lower frequency. The performance of the device, however, is limited by the coupling between the MPP and the backing cavity. By transforming the conventional rectangular cavity to an irregular shaped cavity [4], the coupling can be modified and manipulated due to the distortion of the acoustic mode by tilting cavity walls. The change of the cavity geometry promotes more acoustic modes into the coupling and thereby improves the sound absorption performance at selected frequency bands.

The trend of using MPP in more complex system or in the real industrial setting brings about one critical issue to be addressed. In fact, most of the existing works focused on investigating the property of the MPP device itself, usually validated in a Kundt tube. When placed in a practical environment, however, experimental methods usually become the only option [5] \& [6]. More importantly, the efficiency of the MPP is shown to be influenced by the vibro-acoustic behaviour of the surrounding systems as well as excitations, which may be 
significantly different from the Kundt tube setting. Therefore, the increasing complexity of the system calls for efficient tools to predict the performance of the MPP in a complex vibroacoustic environment. Versatility, efficiency and flexibilities are among the top of the list of major attributes required for such simulation tools, which unfortunately are still lacking in the literature.

This paper attempts to propose a method based on the Patch Transfer Function (PTF) approach for modeling the MPP behaviour in a practical acoustic environment. To illustrate the idea, a general complex vibro-acoustic environment of the MPP is schematically represented in Fig.1(a). Subject to acoustic or mechanical excitations, the whole system is composed of acoustic cavities, semi-infinite acoustic domain, absorbing materials, and flexible panels which are coupled through surfaces. PTF [7] is a substructuring approach which allows assembling different vibro-acoustic subsystems through coupled surfaces. In the present case, the global system is divided into different sub-systems as shown on figure 1 (b). Each coupling surface connecting a pair of subsystem is further divided into elementary areas called patches. The transfer functions, called Patch Transfer Functions (PTFs), of each uncoupled subsystem patch are calculated to form a database. For a mechanical structure, the PTFs are defined as the ratio of the mean velocity over the mean force on a patch, equivalent to patch structural mobilities. For an acoustic domain, the PTFs are defined as the ratio of the mean pressure over the mean velocity on a patch, which are the patch acoustic impedances. Using the superposition principle for linear systems and the continuity relation among different subsystems, the PTF approach allows calculating the response of a global system from the PTFs of uncoupled subsystems by inverting a square symmetric matrix whose dimension corresponds to the number of patches.

The PTFs can be calculated using different methods depending on the subsystem considered. For cavities or flexible structures, these PTFs can be obtained from modal expansions for academic cases or from Finite Element simulation for complex cases. For semi-infinite acoustic domains, Rayleigh integral may be used for a plane boundary or Boundary Element Method (BEM) for a more complex geometry. The sound absorption material may be directly taken into account by its surface impedance. These calculations have been developed and validated for different applications [7-9]. It should be noted that, calculations of PTFs are performed beforehand for each subsystem separately. As a result, parallel computation is possible. Moreover, when FEM is used, the size of the numerical 
models of each subsystem is considerably smaller than that of the global model. In a typical design problem, re-calculations of PTFs are required only for those subsystems or components with modifications, endowing the method the flexibility and efficiency in dealing with complex system, conducive to conducting system optimization.

In this paper, a new formulation for calculating the PTFs of MPP is first proposed. In a first step, the equations of motion of the MPP are expressed on each patch of the coupling surface. The patch flexural velocity of the MPP and the patch acoustic velocity of the surrounding acoustic medium are then linked to the difference of the patch pressures on both sides of the MPP. Two approaches are then proposed: The first one consists in resolving directly the global problem using the MPP relation as a coupling condition with other subsystems. In this formulation, acoustic and mechanical PTFs of each individual subsystem intervene directly in the global equations. Upon casting micro-perforations and the flexibility of the MPP under PTF framework, the proposed PTF formulation provides explicit representation of the coupling between subsystems and facilitates explanation of physical phenomenon. The second approach consists in first calculating the equivalent Patch Transfer Functions of a MPP with a backing cavity, which can be further coupled with the PTFs of other fluid domains in a second step. This second formulation is then explored to illustrate the application of the proposed model to a cavity-backed MPP absorber with an infinite baffle. This allows a deep analysis on the coupling between the MPP and the backing cavity and a quantification of their effects on the sound absorption. It is shown that a MPP with the backing cavity does not behave like a locally reactive material, especially at resonances of the backing cavity. Finally, the proposed model is validated through comparisons with experimental results given in the literature [10].

\section{Principle of PTF approach}

Let us consider the basic vibro-acoustic problem presented in Fig. 2 corresponding to a thin elastic structure coupled on both side with an acoustic domain. The acoustic domain may either be closed or semi-infinite. The PTF approach is briefly recalled here based on this basic system for the sake of clarity, bearing in mind that the methodology can be extended to more complex linear systems. 
Assuming harmonic excitations at an angular frequency $\omega$, we are interested in the steady response of the system, such omitting the time dependence in the notation. Along the surface $S_{c}$ occupied by the thin structure, the whole system is partitioned into three subsystems: an elastic structure and the two acoustic domains at each side. The coupling surface $S_{c}$ is then divided into $N$ elementary surfaces $\partial S_{i}, i \in[1, N]$, called patches. The size of the patches should be less than the half wavelength (i.e. $\lambda / 2$ ) corresponding to the highest frequency of interest, either acoustic or structural, whichever is less [7, 9].

The Patch Transfer Functions (PTFs) are defined for each subsystem, with all quantities being defined with respect to the unit normal vector $\vec{n}$ to the coupling surface $S_{c}$. For the structure, a constant normal force $\bar{f}_{i}^{s}$ is prescribed on patch $i$, whereas no force is prescribed on the other patches. The PTFs between the two patches, ${ }^{Y_{i j}}$, is defined as the ratio between the mean normal velocity on patch $j$ and the normal force $\bar{f}_{i}^{s}$ : $Y_{i j}^{s}=\frac{\bar{u}_{j}^{s}}{\bar{f}_{i}^{s}}$

where $\bar{u}_{j}^{s}$ is the space-averaged normal velocity on the patch, i.e. $\bar{u}_{j}^{s}=\frac{1}{\partial S_{j}} \int_{\partial S_{j}} u_{j}^{s} d S$,

The afore-defined PTFs are equivalent to structural motilities of the structure.

For each acoustic domain $\alpha(\alpha=[1,2])$, a constant normal velocity $\bar{u}_{i}^{\alpha}$ is imposed on patch $i$ with a null normal velocity on the other patches. The PTFs between excited patch $i$ and receiving $\operatorname{patch} j, Z_{i j}^{\alpha}$, is defined as:

$Z_{i j}^{\alpha}=\frac{\bar{f}_{j}^{\alpha}}{\bar{u}_{i}^{\alpha}}$

where $\bar{f}_{j}^{\alpha}$ is the resulted force from the acoustic pressure $p^{\alpha}$ on the patch $j$ when patch $i$ $\underset{f_{j}^{\alpha}}{\text { vibrates with }^{\alpha}} \int_{\partial S_{j}} p^{\alpha} d S$ 
The PTFs such defined correspond to the conventional acoustic impedance.

For the above definitions, the bar in the notation indicates the mean velocities or the sum of the pressure on the patches (i.e. Eqs. $(2,4)$ ). To alleviate the notations, these bars are suppressed in the following.

The coupling between the structure and the acoustic domains is performed in two steps:

- The first step consists in using the linearity properties of the system to express the relationship between the patch velocities and the patch forces for each subsystem. Indeed, the patch velocity of the structure can be expressed as a sum of the velocity due to the mechanical force acting on the structure before coupling $\tilde{u}_{i}^{s}$ and the velocities resulted from the forces exerted on each patch:

$u_{i}^{s}=\tilde{u}_{i}^{s}+\sum_{j=1} Y_{i j}^{s} f_{j}^{s}, \forall i \in[1, \ldots, N]$

Similarly, the force on a patch is equal to the sum of the force corresponding to the acoustic source with a rigid surface, ${ }_{i}{ }^{\alpha}$, and the forces generated by the patch vibrations:

$$
\begin{aligned}
& f_{i}^{1}=\tilde{f}_{i}^{1}+\sum_{j=1}^{N} Z_{i j}^{1} u_{j}^{1}, \forall i \in[1, \ldots, N] \\
& f_{i}^{2}=\tilde{f}_{i}^{2}+\sum_{j=1}^{N} Z_{i j}^{2} u_{j}^{2}, \forall i \in[1, \ldots, N]
\end{aligned}
$$

- the second step consists in writing the continuity conditions at each connecting patch, namely the force equilibrium and the equality of normal velocities:

$$
\begin{aligned}
& u_{i}^{1}=u_{i}^{2}=u_{i}^{s}, \forall i \in[1, \ldots, N] \\
& f_{i}^{s}=f_{i}^{1}-f_{i}^{2}, \forall i \in[1, \ldots, N]
\end{aligned}
$$

where it was assumed that the normal vector is from the acoustic domain 1 toward 2.

$$
\begin{aligned}
& \text { Introducing (5), (6) into (7) } \text { pields }_{N} \\
& u_{i}^{1}=\tilde{u}_{i}^{s}+\sum_{j=1} Y_{i j}^{s}\left(\tilde{f}_{j}^{1}-\tilde{f}_{j}^{2}\right)+\sum_{j=1} Y_{i j}^{s}\left[\sum_{k=1}^{1}\left(Z_{j k}^{1}-Z_{j k}^{2}\right) u_{k}^{1}\right], \forall i \in[1, \ldots, N]
\end{aligned}
$$

This system of linear equations with ${ }^{1}$ as unknowns may be written in the matrix form: 
$\mathrm{u}^{1}=\tilde{\mathrm{u}}^{s}+\mathrm{Y}^{s}\left(\tilde{\mathrm{f}}^{1}-\tilde{\mathrm{f}}^{2}\right)+\mathrm{Y}^{s}\left(\mathrm{Z}^{1}-\mathrm{Z}^{2}\right) \mathrm{u}^{1}$

Equation (9) is a full system, with its size being equal to the number of patches. Upon resolving this system,

$\mathrm{u}^{1}=\left[\mathrm{I}-\mathrm{Y}^{s}\left(\mathrm{Z}^{1}-\mathrm{Z}^{2}\right)\right]^{-1}\left[\tilde{\mathrm{u}}^{s}+\mathrm{Y}^{s}\left(\tilde{\mathrm{f}}^{1}-\tilde{\mathrm{f}}^{2}\right)\right]$

where $\mathrm{I}$ is the $N \times N$ identity matrix .

All other physical quantities, such as acoustic pressure in each domain can be calculated in a post-processing phase.

The PTF approach allows calculating the response of a global system from the PTFs of uncoupled subsystems by inverting a square symmetrical matrix whose dimension corresponds to the number of patches. The PTFs can be calculated by different methods depending on the subsystem considered. These calculations are performed beforehand for each subsystem separately. When FEM is used, the size of the numerical models of each subsystem is considerably smaller than that of the global model. Moreover, the use of incompatible meshes at the subsystem interface is possible, since the problem of compatibility is solved by patch averaging.

\section{PTF equations for the micro-perforated structure subsystem}

Assuming the structure separating the two acoustic domains in Fig.2 takes the form of a micro-perforated structure, corresponding PTF equations will be developed in the following sections.

\subsection{Modeling of micro-perforated structure}

Considering a MPP element shown in Fig.3, the sound pressure difference between the two sides of MPP, $p^{1}-p^{2}$ generates the vibration of air mass $u^{0}$ at each single hole. As the orifice diameter of the hole is much smaller than the acoustic wavelength of interests, it is 
appropriate to assume that the air particle velocity is distributed uniformly within the area of each hole. Let $Z_{0}$ denote the complex acoustic impedance of the hole normalized by the characteristic impedance $\rho_{0} c_{0}$, where $\rho_{0}$ is the air density and $c_{0}$ the speed of sound. $Z_{0}$ is given by [11, 12]
\[ Z_{0}=\frac{32 \eta t}{\rho_{0} c_{0} d^{2}}\left[\left(1+\frac{K^{2}}{32}\right)^{0.5}+\frac{\sqrt{2}}{32} K \frac{d}{t}\right]+i \cdot \frac{\omega t}{c_{0}}\left[1+\left(1+\frac{K^{2}}{32}\right)^{-0.5}+0.85 \frac{d}{t}\right] \]

where $\omega$ is the angular frequency; $t$ the thickness of the structure; $d$ the orifice diameter; $\eta$ the coefficient of viscosity and, $K=d \sqrt{\frac{\omega \rho_{0}}{4 \eta}}$. The real part is the resistive term which corresponds to the viscous force whereas the imaginary part is the reactance term corresponding to the inertial force.

Assume that the MPP is flexible and let ${ }^{u^{s}}$ be its normal velocity. The viscous force depends on the relative velocity of the air in the hole and the structure, $u^{0}-u^{s}$ and the inertial force depends only on the air velocity. Thus, one can write $[12,13]$ :

$$
\operatorname{Re}\left\{Z_{0}\right\}\left(u^{0}-u^{s}\right)+i \operatorname{Im}\left\{Z_{0}\right\} u^{0}=\frac{1}{\rho_{0} c_{0}}\left(p^{1}-p^{2}\right)
$$

As the orifice diameter of the hole is much smaller than the acoustic and flexural wavelengths of interests, the mean velocity of the surrounding air particle in the vicinity of the MPP, $u^{1}$, can be approximated from the relation:

$$
u^{1}=(1-\sigma) u^{s}+\sigma u^{0}
$$

$u^{1}$ allows expressing the velocity continuity conditions of the MPP with the adjacent acoustic domains. On the contrary, $u^{0}$ will not be directly wsed and can be substituted from Eq. (12):

$$
u^{0}=\frac{1}{\rho_{0} c_{0} Z_{0}}\left(p^{1}-p^{2}\right)+\frac{\operatorname{Re}\left\{Z_{0}\right\}}{Z_{0}} u^{s} \text {. }
$$

Using the above expression in Eq. (13) gives

$$
u^{1}=\left[(1-\sigma)+\frac{\sigma \operatorname{Re}\left\{Z_{0}\right\}}{Z_{0}}\right] u^{s}+\frac{\sigma}{\rho_{0} c_{0} Z_{0}}\left(p^{1}-p^{2}\right)
$$

This relation can be rewritten in a compact form as 


$$
u^{1}=\mathrm{T} u^{s}+\Psi\left(p^{1}-p^{2}\right)
$$

where:

$$
\mathrm{T}=(1-\sigma)+\frac{\sigma \operatorname{Re}\left\{Z_{0}\right\}}{Z_{0}} \text { is a non-dimensional parameter which may be called the MPP }
$$

transmissibility, which represents the contribution of the structure vibrations to the surrounding acoustic particle vibrations.

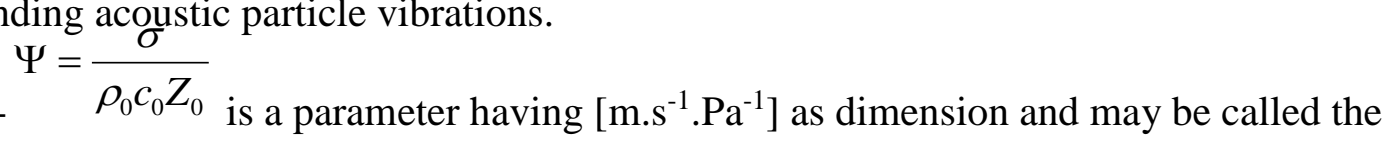

equivalent mobility of the perforation.

Equation (16) clearly demonstrates the underlying relationship among the acoustic velocity of the surrounding medium, the pressure difference across the MPP and the vibration of the structure. The resultant velocity in the vicinity of the MPP is a combination of partial transmission of the structural vibration and the air motion of the micro-perforation. The development of the PTF equation for a MPP subsystem in the next section is based on this relation.

\subsection{PTF developments}

\subsubsection{Direct formulation}

For a MPP patch, whilst Eqs (5-6) relating the patch pressures and the patch velocities remain valid, the continuity conditions at connecting patches need to be modified. Indeed, by a space averaging on the patch $i$ of Eq. (16), one has:

$$
u_{i}^{1}=u_{i}^{2}=\mathrm{T} u_{i}^{s}+\Psi\left(p_{i}^{1}-p_{i}^{2}\right), \forall i \in[1, \ldots, N] \text {. }
$$

Meanwhile, the pressure difference $p_{i}^{1}-p_{i}^{2}$ acting on the $(1-\sigma) \partial S_{i}$ surface of the MPP generates a force, $f_{i}^{s}$, at the patch $i$ :

$$
f_{i}^{s}=\left(p_{i}^{1}-p_{i}^{2}\right)(1-\sigma) \partial S_{i}, \forall i \in[1, \ldots, N]
$$

As $f_{i}^{\alpha}=p_{i}^{\alpha} \partial S_{i}$, these two relations can be rewritten as:

$$
u_{i}^{1}=u_{i}^{2}=\mathrm{T} u_{i}^{s}+\Psi_{i}\left(f_{i}^{1}-f_{i}^{2}\right) \text {, with } \Psi_{i}=\frac{\Psi}{\partial S_{i}}, \quad \forall i \in[1, \ldots, N]
$$




$$
f_{i}^{s}=\left(f_{i}^{1}-f_{i}^{2}\right)(1-\sigma), \forall i \in[1, \ldots, N]
$$

The above expressions describe the continuity conditions in the presence of a microperforated structure.

Introducing the linear decompositions Eqs.(5-6) in Eqs.(19-20), the patch velocity of the fluid domain 1 can be written as:

$$
\begin{array}{r}
\begin{array}{r}
u_{i}^{1}=\mathrm{T} \tilde{u}_{i}^{s}+\sum_{j=1}^{N}\left(\Psi_{i} \delta_{i j}+\mathrm{T}(1-\sigma) Y_{i j}^{s}\right)\left(\tilde{f}_{j}^{1}-\tilde{f}_{j}^{2}\right)+\sum_{j=1}^{N}\left[\left(\Psi_{i} \delta_{i j}+\mathrm{T}(1-\sigma) Y_{i j}^{s}\right) \sum_{k=1}^{N}\left(Z_{j k}^{1}-Z_{j k}^{2}\right) \boldsymbol{u}_{k}^{1}\right] \\
, \forall i \in[1, \ldots, N]
\end{array}
\end{array}
$$

where $\delta_{i j}$ is the Kronecker symbol. The above system may be condensed into a matrix form:

$$
\mathrm{u}^{1}=\mathrm{T} \tilde{\mathrm{u}}^{s}+\left(\Psi+\mathrm{T}(1-\sigma) \mathrm{Y}^{s}\right)\left(\tilde{\mathrm{f}}^{1}-\tilde{\mathrm{f}}^{2}\right)+\left(\Psi+\mathrm{T}(1-\sigma) \mathrm{Y}^{s}\right)\left(\mathrm{Z}^{1}-\mathrm{Z}^{2}\right) \mathrm{u}^{1}
$$

which admits solution in the following form:

$$
\mathrm{u}^{1}=\left[\mathrm{I}-\left(\Psi+\mathrm{T}(1-\sigma) \mathrm{Y}^{s}\right)\left(\mathrm{Z}^{1}-\mathrm{Z}^{2}\right)\right]^{-1}\left[\mathrm{~T} \tilde{\mathrm{u}}^{s}+\left(\Psi+\mathrm{T}(1-\sigma) \mathrm{Y}^{s}\right)\left(\tilde{\mathrm{f}}^{1}-\tilde{\mathrm{f}}^{2}\right)\right]
$$

Note $\Psi$ is a diagonal matrix. In a post-processing phase, the pressure inside the acoustic domains and the velocity on the MPP can be calculated..

Equation (23) seems to differ significantly from Eq. (10). However, if we introduce an equivalent PTFs, ${ }^{e q}$ and equivalent free patch velocities, ${ }^{e q}$ such that:

$$
\mathrm{Y}^{e q}=\Psi+\mathrm{T}(1-\sigma) \mathrm{Y}^{s} \text {, and, } \tilde{\mathrm{u}}^{e q}=\mathrm{T} \tilde{\mathrm{u}}^{s}
$$

one can rewrite Eq. (23) as:

$$
\mathrm{u}^{1}=\left[\mathrm{I}-\mathrm{Y}^{e q}\left(\mathrm{Z}^{1}-\mathrm{Z}^{2}\right)\right]^{-1}\left[\tilde{\mathrm{u}}^{e q}+\mathrm{Y}^{e q}\left(\tilde{\mathrm{f}}^{1}-\tilde{\mathrm{f}}^{2}\right)\right]
$$

This expression takes the same form as Eq. (10) except that the PTFs and patch free velocities are replaced by their equivalent expressions for the MPP case.

In the case of a rigid MPP (i.e. $\mathrm{Y}^{s}=0$ and $\tilde{\mathrm{u}}^{s}=0$ ), Eq. (23) becomes:

$$
\mathrm{u}^{1}=\left[\mathrm{I}-\Psi\left(\mathrm{Z}^{1}-\mathrm{Z}^{2}\right)\right]^{-1} \Psi\left(\tilde{\mathrm{f}}^{1}-\tilde{\mathrm{f}}^{2}\right)
$$


and the equivalent PTFs and patch free velocity of the MPP are:

$$
\mathrm{Y}^{e q}=\Psi \text {, and, } \tilde{\mathrm{u}}^{e q}=0 \text {. }
$$

Then, the equivalent PTFs are equal to the MPP mobility for the input terms (i.e. $\mathrm{Y}_{i i}^{e q}=\Psi_{i}, \forall i \in[1, \ldots, N]$ ) and are null for the cross terms (i.e $\mathrm{Y}_{i j}^{e q}=0, i \neq j$ ). In this case, the MPP can be considered as a locally reactive structure characterised by the mobilities $\Psi$. On the contrary, as can be shown later, a MPP with a backing cavity cannot be considered as a locally reactive device, as opposed to the common assumption made for porous absorbing materials.

\subsubsection{Equivalent PTFs for a cavity-backed MPP}

A typical micro-perforated panel absorber takes the form of a MPP fitted in front of a backing wall or a cavity. The air gap/volume behind the MPP provides an acoustic-stiffness [4] which leads to resonance-type absorption with the perforation. This cavity-backed MPP finds its use in various system configurations and therefore deserves a particular treatment. In order to facilitate the modeling of the overall system, a cavity-backed MPP can be regarded as a standalone subsystem. Once the acoustic property is known in terms of PTFs over its surface, it can be integrated into the conventional PTF framework [7], such providing an alternative to the direct formulation presented in Section 3.2.1. Meanwhile, this will allow a significant simplification and the down-sizing of the number of the sub-system to be handled in a complex system.

To this end, the equivalent PTF of a cavity-backed MPP is defined, to be obtained according to the calculation scheme established hereafter. Consider a typical MPP backed by an acoustic cavity. The outer surface $S_{c}$ of the cavity-backed MPP is divided into $N$ patches. By imposing a unit normal velocity on patch $i$, the resulted force on patch $j$ needs to be calculated using the PTFs of the cavity and the MPP.

The same matrix notation as in the previous section is used and a $\boldsymbol{\Xi}$ vector is defined as:

$$
\boldsymbol{\Xi}=\left[\Xi_{k}\right]_{1 \times N} \quad \Xi_{k}=\left\{\begin{array}{l}
1, \text { for } k=l, \\
0, \text { otherwise. }
\end{array}\right.
$$


The resulted forces on the patches are contained in the $\mathrm{f}^{1}$ vector and the imposed velocity condition writes:

$$
\mathrm{u}^{1}=\Xi
$$

The continuity relations Eq (19) and Eq. (20) become:

$$
\begin{gathered}
\mathrm{u}^{2}=\Xi, \text { and, } \\
\mathrm{T} \mathrm{u}^{s}+\Psi\left(\mathrm{f}^{1}-\mathrm{f}^{2}\right)=\Xi
\end{gathered}
$$

As the MPP and the cavity are not directly excited, Eqs.(5-6) become

$$
\begin{aligned}
& \mathrm{u}^{s}=\mathrm{Y}^{s} \mathrm{f}^{s} \\
& \mathrm{f}^{2}=\mathrm{Z}^{2} \mathrm{u}^{2}
\end{aligned}
$$

Combining Eqs.(30-33) yields

$$
\left(\Psi+\mathrm{T}(1-\sigma) \mathrm{Y}^{s}\right)\left(\mathrm{f}^{1}-\mathrm{Z}^{2} \Xi\right)=\Xi
$$

The resulting forces ${ }^{1}$ on the patches due to a unit velocity imposed on patch $i$ write

$$
\mathrm{f}^{1}=\left[\left[\Psi \mathrm{I}+\mathrm{T}(1-\sigma) \mathrm{Y}^{s}\right]^{-1}+\mathrm{Z}^{2}\right] \Xi
$$

This gives the equivalent PTF between patch $i$ and patch $j, \forall j \in[1, N]$. One finally deduces the equivalent PTF matrix:

$$
\mathrm{Z}^{\mathrm{eq}}=\left[\Psi \mathrm{I}+\mathrm{T}(1-\sigma) \mathrm{Y}^{s}\right]^{-1}+\mathrm{Z}^{2}
$$

These equivalent PTFs may be used in the classical PTF approach to represent the behaviour of the MPP with a backing cavity. In such case, the standard continuity relations Eqs (7) should be used to assemble the equivalent PTFs with the PTFs of the connected subsystem. Eq. (36) also gives indications on the behaviour of the system and in particular, on whether the system has a localised reaction. With a rigid MPP, the equivalent PTFs become:

$$
\mathrm{Z}^{\mathrm{eq}}=\Psi^{-1}+\mathrm{Z}^{2}
$$

where $\Psi^{-1}$ is a diagonal matrix. The system is locally reactive if the first term dominates (i.e. $\mathrm{Z}^{\mathrm{eq}} \approx \Psi^{-1}$ ). Otherwise, the system is not locally reactive when $\mathrm{Z}^{\mathrm{eq}}$ is full due to the cavity 
effect characterized by $Z^{2}$. Examples will be given in the next section for further elaborate this point.

\section{An illustration of application}

The general framework of a MPP coupled to a complex environment is now established by using the PTF approach. In this section, the proposed method is applied to a basic case which differs from the conventional case of a micro-perforated panel in an acoustic tube. The purpose is to show the potential of the present approach, instead of detailed treatment of the configuration itself.

\subsection{A cavity-backed micro-perforated panel inserted in a rigid baffle}

Let us consider a MPP backed by rectangular cavity. The surface of the MPP is flush with a rigid baffle of infinite size. Excited by an incident plane wave $p_{0} e^{j\left[k_{0} \sin (\theta) x-k_{0} \cos (\theta) z\right]}$ impinging on the MPP at an angle of incidence ${ }^{\theta}$, the MPP radiates sound towards both the cavity and the semi-infinite acoustic domain. For an easier interpretation of the results, the MPP is supposed to be rigid, bearing in mind that this assumption is not a limitation of the approach as the structure vibration has already been incorporated into the PTF model. Previous investigations demonstrated that the panel vibration mainly affects the absorption performance at the structural resonances [13]. The size of the panel is $0.5 \mathrm{~m} \times 0.5 \mathrm{~m}$ whereas the depth of the cavity is $0.3 \mathrm{~m}$. The panel thickness, $t$ and the orifice diameter, $d$ are both $0.2 \mathrm{~mm}$. The perforation ratio $\sigma$ is $1 \%$. The acoustic medium involved is air ( $\rho_{0}=1.29 \mathrm{~kg} / \mathrm{m}^{3}$, $c_{0}=340 \mathrm{~m} / \mathrm{s}$ ). The first 24 modes of the rigid-walled cavity are tabulated in Table 1.

\subsection{PTF calculation procedure}

The PTF approach presented in this paper is used for estimating the acoustic pressure in the semi-infinite medium and the absorption coefficient of the MPP with the backing cavity. We are interested in the response of this system in a frequency range up to $2000 \mathrm{~Hz}$. The system is decomposed into three PTF subsystems: the MPP, the backing cavity and the infinite acoustic 
medium. We are typically in the situation described in section 3 with an excitation in the domain 1 which is semi-infinite. The coupling surface between the three PTF subsystems is divided in 81 patches ( $N=9 \times 9=81$ ) ensuring a patch size less than half the acoustic wavelength at $2000 \mathrm{~Hz}$ (in accordance with the patch size criterion $\lambda / 2$ defined in [7]). The PTFs of the cavity are calculated using a modal expansion method as detailed in Appendix A (A.1). Acoustic modes of the rigid-walled cavity having a natural frequency below $4000 \mathrm{~Hz}$ are used to ensure the convergence of the calculation. For the semi-infinite domain, the PTFs are estimated by using the Rayleigh integral as described in appendix A.2. It is relevant to note that they are independent of the incident wave and of the characteristics of the MPP.

As the MPP is impinged by an incident plane wave propagating in the semi-infinite medium, the patch blocked forces of the acoustic domain $1, \tilde{f}_{i}^{1}$ are:

$$
f_{i}^{1}=2 \int_{\partial S_{i}} p_{\text {inc }}(M) d S, \forall i \in[1, \ldots, N]
$$

where $p_{\text {inc }}(M)=p_{0} e^{j\left[k_{0} \sin (\theta) x-k_{0} \cos (\theta) z\right]}$, and the patch blocked forces of the fluid domain 2 (i.e. cavity), $\tilde{f}_{i}^{2}$ are:

$$
\tilde{f}_{i}^{2}=0, \forall i \in[1, \ldots, N]
$$

For a normal incident wave, $\theta=0$, one has:

$$
\tilde{f}_{i}^{1}=2 p_{0} \partial S_{i} \text { and } \tilde{f}_{i}^{2}=0, \forall i \in[1, \ldots, N]
$$

Using these patch blocked forces and the PTFs of the cavity and the semi-infinite medium, one can calculate the patch velocities, $u_{i}^{1}, \forall i \in[1, \ldots, N]$ from Eq. (23) when the MPP is coupled with the backing cavity on one side and the semi-infinite medium on the other side. The acoustic power absorbed by the cavity-backed MPP, $\Pi_{a b s}$ can then be derived from:

$$
\Pi_{a b s}=\frac{1}{2} \sum_{i=1}^{N} p_{i}^{1}\left(u_{i}^{1}\right)^{*} \partial S_{i}
$$

where the asterisk denotes the complex conjugate. The patch pressures, $p_{i}^{1}$ can be calculated from:

$$
p_{i}^{1}=\frac{1}{\partial S_{i}}\left(\tilde{p}_{i}^{1}+\sum_{j=1}^{N} Z_{i j}^{1} u_{j}^{1}\right)
$$


The absorption coefficient $\alpha_{a b s}$ is defined as the ratio of the absorption power over the incident power through the MPP surface:

$$
\alpha_{a b s}=\frac{\Pi_{a b s}}{\prod_{i n c}}
$$

where $\Pi_{i n c}$ is the acoustic power injected on the MPP surface by the incident plane wave propagating freely in the acoustic medium:

$$
\Pi_{i n c}=\frac{1}{2} \frac{p_{0}{ }^{2}}{\rho_{0} c_{0}} \sum_{i=1}^{N} \partial S_{i} \text {. }
$$

A patch absorption coefficient can be defined for each patch (i.e. local absorption coefficient) as the ratio of the absorption power by the incident power through the patch surface:

$$
\alpha_{a b s, i}=\frac{\rho_{0} c_{0}}{p_{0}{ }^{2}} p_{i}^{1}\left(v_{i}^{1}\right)^{*}, \forall i \in[1, \ldots, N] \text {. }
$$

These coefficients indicate the distribution of the absorption through the MPP surface. In the case where all patches are identical,

$$
\alpha_{a b s}=\frac{1}{N} \sum_{i=1}^{N} \alpha_{a b s, i}, \forall i \in[1, \ldots, N] .
$$

\subsection{Analysis of results}

\subsubsection{Normal incident wave}

The results show in this section concern an incident plane wave, normal to the MPP surface and having unit amplitude ( $p_{0}=1 \mathrm{~Pa}$ ). As can be seen from section 2 , although the oblique incident wave excitation is not a limitation of the current model, we stick to the normal incident case so that results can be compared with literature.

\subsubsection{Absorption coefficient}

The sound absorption coefficient is calculated using the PTF formulation described in section 4.2. Numerical results are compared with the results of the equivalent electric circuit method in Fig.4. Note the electric circuit method considers an infinite MPP with an air gap of the same depth in one dimensional case. With a limited panel size, the three dimensional system 
considered in the present PTF scheme differs significantly from the classical one dimensional case. Figure 4 shows that the two methods give similar results in the high frequency range whereas significant differences occur at low frequencies. At some frequencies, PTF results give a sound absorption coefficient greater than one for our system. This behaviour can be explained by the fact that the present system is three dimensional one, in which the "edge effect" [14-18] occurs. In the low frequency range, the diffraction phenomenon in the vicinity of the edge of the MPP results in an increase of the energy absorption coming from the neighbourhood of the edges. This can be better seen by investigating the acoustic intensity in the neighbourhood of the MPP. The calculation of the acoustic intensity in the framework of the PTF is described in Appendix B. The acoustic intensity maps are then plotted for three selected frequencies corresponding to the local maxima (of Fig.4) in figure 5. One can clearly observe that at $150 \mathrm{~Hz}$, corresponding to the maximum absorption, the energy entry into the MPP comes not only from the front of the MPP due to the acoustic incidence, but also from the side way of the MPP (due to the diffraction effect). As the definition of the absorption coefficient only considers the acoustic power of the incident wave over a surface corresponding to the MPP surface (see Eq. 43), the acoustic power diffracted from the side of the MPP is not taken into account. This results in an underestimation of the actual power impinging on the panel and an absorption coefficient greater than one in the low frequency range. For higher frequencies, Fig. 5 shows the absorbed power predominately comes from the front and the effect of the diffraction becomes more and more negligible when frequency increases. This can also be reflected in Fig.4 in which the corresponding sound absorption coefficient is lower than one.

Based on this understanding, one can surmise that the size of the MPP with respect to the acoustic wave length should play an important role in this phenomenon. This was indeed shown in the past by different authors considering classical absorbing material of finite sizes [15-18]. They showed that the absorption coefficient of a patch of absorbent material depends on the size of the patch relative to the wavelength. In general, the absorption coefficient increases when the size of the patch material decreases. Same phenomenon has been observed in the present MPP case, in agreement with the previous studies [15-18]. It should be noted however that these studies considered porous or fibrous absorbing materials which present rather poor absorption at low frequencies. On the contrary, depending on its design, cavitybacked MPP provides efficient sound absorption even at relatively low frequencies, where the 
acoustic wavelength is large. By the same token, the size effect is also reinforced in the case of a MPP.

By increasing the MPP size to $2 \mathrm{~m} \times 2 \mathrm{~m}$, we approach more closely to the infinite panel scenario which the equivalent circuit method can model. Results obtained form the current PTF approach is superposed to the two existing curves in Fig.4. It can be seen that using a larger panel, the present PTF approach gives similar result as the equivalent circuit method does, such demonstrating the validity of the approach.

The spatial distribution of the energy absorption by the MPP at the same frequencies can be revealed using the patch absorption coefficients defined by Eq. (45), shown in Fig.6. At 150 $\mathrm{Hz}$, the absorption is rather uniformly distributed which may be explained by the large wavelength $(2.26 \mathrm{~m})$ at this frequency. At $707 \mathrm{~Hz}$, corresponding to the second peak in the absorption coefficient curve, the patch absorption coefficients are significantly greater on the boundaries of the MPP than the centre, reaching maximum around the corners. This result can be expected given that the diffraction phenomenon occurs at the boundary and the acoustic wavelength is of the same order of magnitude as the panel size. For an even higher frequency at 1267 , one observes variations of the patch absorption coefficients with some values greater than one whereas the (global) absorption coefficients remain lower than one. The diffraction phenomenon in this $3 \mathrm{D}$ system and the size of the acoustic wavelength (i.e. $0.27 \mathrm{~m}$ ) may explain this distribution.

\subsubsection{Equivalent Patch Transfer Functions}

The proposed PTF formulation provides explicit representation of the coupling between subsystems and facilitates explanation of physical phenomenon. This feature is explored using the current system of cavity-backed MPP in terms of the equivalent PTFs defined in Section 3.3.2.

The equivalent PTFs are calculated and depicted in Fig.7 for three different frequencies. Figure 7(a) shows that at $150 \mathrm{~Hz}$, the equivalent PTF matrix, ${ }^{\text {eq }}$ (Eq. (37)) is a diagonaldominant matrix and $Z^{2}$ in Eq. (37) is negligible comparing with $\Psi^{-1}$. This shows that the system is rather local reactive at this frequency. This gives, 


$$
\mathbf{Z}^{\text {eq }} \approx \frac{\partial S}{\Psi} \mathbf{I}
$$

where $\partial S$ is the surface of the patches. At this frequency, the equivalent PTFs depend only on the equivalent mobility of the perforation $\Psi$ and the surface of the patches, indicating the strong dominance of the MPP. On the contrary, for the two other frequencies corresponding to the first two modes of the hard-walled cavity, the equivalent PTF matrices are full matrices as shown in Fig.7(b-c), which indicates the system has not a localised reaction. In this case, the behaviour of the cavity-backed MMP is dominated by the cavity. The equivalent PTFs vary in function of the modal variation on the coupling surface. As an extreme example, from the third modes $(0,0,1)$ at $567 \mathrm{~Hz}$, the PTFs are quasi-constant as shown in Fig.7(c) due to the uniform spatial shape of this mode on the coupling surface. Slight variations at the diagonal terms are due to the term $\frac{\partial S}{\Psi}$ in Eq. (37).

It is pertinent to mention that $\frac{\partial S}{\Psi}$ is the impedance of the perforation and becomes the impedance of the MPP when the vibration of the latter is neglected. The comparison between this impedance term and the impedance of the cavity provides very useful physical insight in the way the MMP is coupled to the cavity. This is done in Fig.8, in which the impedance of the MPP, $\frac{\partial S}{\Psi}$, is compared to that of the cavity $Z_{i i}$ for one particular patch $(3,4)$. It can be seen that, $\frac{\partial S}{\Psi}$ are generally larger than $Z_{i i}$, except for frequencies close to the natural frequencies of the cavity. Provided these modes are excited, the equivalent PTFs correspond to the cavity impedance at their resonance frequencies. In that case, the effect of the MPP becomes negligible compared to the cavity resonance. One such example is the mode $(0,0,1)$ at $567 \mathrm{~Hz}$. As the acoustic damping inside the cavity is small $(\eta=0.0001)$, the absorption coefficient is quasi null at this frequency, as shown in Fig.4. Similar observations can be made at $1133 \mathrm{~Hz}$ and $1700 \mathrm{~Hz}$ which correspond to the natural frequencies of modes $(0,0,2)$ and $(0,0,3)$, respectively. It should be mentioned that quite a few acoustic modes cannot be excited due to the nature of the normal incident wave, which are reflected in the sound absorption curve. 
In conclusion, the equivalent PTFs of the cavity-backed MPP allows representing the MPP coupled with the backing cavity as a single PTF subsystem. This subsystem cannot be considered as a locally reactive material in general.

\section{EXPERIMENTAL VALIDATIONS}

The proposed PTF approach is used to investigate the acoustic field inside a rectangular cavity used in Fenech et al. [10]. Results obtained from the present approach are compared with the experimental data reported in that paper. This comparison allows further validation of the proposed approach in a vibro-acoustic environment. The rectangular air cavity of dimension $2 \mathrm{~m} \times 1.2 \mathrm{~m} \times 0.2 \mathrm{~m}$ was made of $22-\mathrm{mm}$ fiberboard panels screwed and glued together. As shown in Fig. 9. a loudspeaker with a diameter of $9 \mathrm{~cm}$ was mounted on one of the vertical side panels to provide a white noise excitation to the cavity. The sound pressure was measured at various locations for three configurations: (a) the empty cavity; (b), a MPP installed at the middle of the cavity at $\mathrm{x}=1 \mathrm{~m}$; (c), a MPP installed near the cavity wall at $\mathrm{d}=0.25 \mathrm{~m}$ (Fig.9). In Fenech et al. [10], the reverberation time of the empty cavity was found to be roughly $0.5 \mathrm{~s}$ in most of the frequency range of interest. This allows to estimate an overall damping loss factor from $\eta=2.2 / f T_{r}$ where $f$ is the frequency and $T_{r}$ the reverberation time. The aluminium MPP was 1-mm-thick and supplied by the Swedish manufacturer Sontech under the trade name Acustimet. Due to the manufacturing process, the punched perforations were found to produce holes with sharp edges that protrude out of the surface of the plate. Therefore, instead of using Maa's theory [11], the flow impedance $Z_{0}$ was experimentally measured using an impedance tube and used in their model [10]. The vibration of the MPP was supposed to be negligible, i.e. $Y_{i j}^{s}=0, \forall(i, j) \in[1, N]^{2}$ in the present formulation.

When the MPP is placed inside the cavity, the system is divided into three subsystems: the MPP, and the two rectangular sub-cavities as shown on Fig. 10. The rectangular coupling surface is divided in 5 patches to ensure the convergence of the solution up to $500 \mathrm{~Hz}$, according to the $\lambda / 2$ criterion. The PTFs of the sub-cavities are calculated from the modal method using rigid-walled modes of the sub-cavities below $1000 \mathrm{~Hz}$ (Appendix A.1). This calculation is the most time consuming part, even though it requires only few seconds in the case. These PTFs are also used for calculating the response of the empty cavity in the PTF 
approach. In this case, the system has only two subsystems, namely the two sub-cavities, connected by a virtual surface between them at the middle of the cavity. Of course, it is straightforward to use modes of the entire cavity in a modal expansion scheme for estimating the response of the empty cavity. The goal of treating the system by PTF approach is to highlight the efficiency of the method through comparisons with experimental measurement.

Comparisons between the present PTF and experimental results obtained by Fenech et al. are made in Fig. 11 in terms of sound pressure level at the measurement point. For the empty cavity, Fig.11 (a) shows a satisfactory agreement between these two sets of results, despite some noticeable differences in the resonance frequencies as well as the peak values. The discrepancy in frequencies may be due to the cavity boundary used in experiment, which does not totally comply with theoretical model. Indeed, the first nonzero natural frequency of the cavity was predicted at $88 \mathrm{~Hz}$ by the present PTF approach, as opposed to $93 \mathrm{~Hz}$ measured experimentally and $86 \mathrm{~Hz}$ calculated from analytical solutions under the assumption of rigid walls. It is remarkable that, although this frequency does not correspond to any of the natural frequencies of the sub-cavities, it was correctly predicted using PTF approach.

With the MPP placed at the middle of the cavity, similar comparisons are plotted in Fig.11 (b). Again, the agreement between the PTF results and the experimental results is globally good. Compared with Fig.11(a), the effect of the MPP can be observed: some resonance frequencies of the empty cavity are altered by the presence of the MPP on one hand, and reduction of some resonance peaks due to the sound absorption of the MPP on the other hand. An attenuation of as much as $25 \mathrm{~dB}$ for some modes (i.e. $(1,0),(3,0),(3,1))$ noticed in the experiment of Fenech et. al.) are well reproduced by our simulations. It is noteworthy that due to the location of the MPP, some modes are practically not affected by the presence of the MPP.

As the last example, Fig. 12 compares the PTF result with that obtained by Fenech' calculation based on the complex mode evaluation of the modified cavity, along with the experimentally measured sound pressure level when the MPP was placed toward a wall of the cavity $d$ $=0.25 \mathrm{~m}$. Despite some differences, the agreement between the experiment and the simulation is generally satisfactory, bearing in mind that the sound pressure level is sensitive to the location of the measurement point. Similar agreement was also observed for other figures 
presented in [10] (not shown here). The two calculation models give very similar results albeit more apparent discrepancies occur at higher frequencies. Differences and uncertainties in the modeling (i.e. damping model, modal convergence) and the data (i.e. damping value, flow impedance value) may explain these differences.

In conclusion, these comparisons demonstrate the validity of the present PTF formulation in dealing with systems of various configurations.

\section{Conclusions}

A vibroacoustic formulation based on the Patch Transfer Functions (PTF) approach is proposed to model micro-perforated structures in a complex vibro-acoustic environment. The PTFs of the micro-perforated structure is first formulated. Its coupling with surrounding acoustic domains is then cast into transfer function paradigm through velocity continuity and force equilibrium over connecting patches. This leads to two different formulations, namely direct formulation and equivalent PTFs for cavity-backed micro-perforated structure, both providing explicit representation of the coupling between subsystems and facilitating physical explanations.

By virtue of its substructuring nature, the PTF approach proposed in this paper is an efficient tool to deal with micro-perforated structure in a complex vibro-acoustic environment. Calculations of PTFs are performed beforehand for each subsystem separately. As a result, parallel computation is possible. In a typical design problem, re-calculations of PTFs are required only for those subsystems or components with modifications, endowing the method the flexibility and efficiency in dealing with complex system, conducive to conducting system optimization.

As an illustration example, application to a MPP with a backing cavity located in an infinite baffle is demonstrated. The proposed PTF formulation is finally validated through comparison with experimental measurements available in the literature.

As a final note, it is relevant to mention that the PTF approach has been applied to several basic systems in this paper. It can be extended to more practical cases with complex geometries. In this case, the patch transfer functions of each subsystem may be calculated by 
numerical methods like Finite Element Method or Boundary Element Method. The size of the numerical models of each subsystem is considerably smaller than that of the global model.

\section{Reference}

[1] Maa D. Y., Theory and design of microperforated-panel sound-absorbing construction, Sci. Sin. Vol. XVIII, 55-71, 1975.

[2] Zhang Z. M. and Gu X. T., The theoretical and application study on a double layer microperforated sound absorption structure, J. Sound Vib. 215, 399-405,1998.

[3] Lee D. H., and Kwon Y. P. Estimation of the absorption performance of multiple layer perforated panel systems by transfer matrix method, J. Sound Vib. 278, 847-860, 2004.

[4] Wang C., Cheng L., Pan J., Ganghua Y.,Sound absorption of a micro-perforated panel backed by an irregular-shaped cavity. J. Acoust Soc Am 2010; 127(1):238-246.

[5] Kang J., and Fuchs H. V., Feasibility of applying microperforated absorbers in acoustic window system, Appl. Acoust. 66, 669-689, 2005.

[6] Droltleff H., and Zhou X., Attractive room acoustics design for multi-purpose halls, Acustica. 87, 500-504,2001.

[7] Ouisse M., Maxit L., Cacciolati C., and Guyader J-L. Patch Transfer Functions : a Tool to Couple Linear Acoustic Problems. J Vibr-Acoust ASME 2005;127:458-466.

[8] Maxit L., Cacciolati C., and Guyader J-L. Airborne noise prediction using patch acoustic impedance. Proceedings of International Congress on Sound and Vibration ICSV9, Orlanda, United States, 2002.

[9] Chazot J-D, Guyader J-L. Prediction of transmission loss of double panels with a patch-mobility method. J Acoust Soc Am 2007;121(1):267-78

[10] B. Fenech, G.M. Keith, F. Jacobsen, The use of microperforated plates to attenuate cavity resonances. J. Acoust Soc Am 2006; 120(4):1851-1858.

[11] Maa D., Potential of microperforated panel absorber, J. Acoust Soc Am 1998; 104(5):2861-2866.

[12] Takahaski D., M. Tanaka, Flexural vibration of perforated plates and porous elastic materials under acoustic loading. J. Acoust Soc Am 2002; 112 (4): 1456-1463. 
[13] Lee Y.Y., Lee E.W.M., Ng C.F., Sound absorption of a finite flexible micro-perforated panel backed by an air cavity. J. Sound and Vib. 2005; 287: 227-243.

[14] Sabine P.E., What is measured in sound absorption measurements. J. Acoust Soc Am 1935; 6: 239-245.

[15] Pellam J.R., Sound Diffraction and absorption by a strip of absorbing material. J. Acoust Soc Am 1940; 11:396-400.

[16] Cook, R.K., Absorption of Sound by Patches of Absorbent Materials. J. Acoust Soc Am 1957; 29(3):324-329.

[17] Pellam J.R., Bolt R.H., The absorption of Sound by Small Areas of Absorbing Material. J. Acoust Soc Am 1940; 12:24-30.

[18] Daniel. E.D., On the dependence of absorption coefficients upon the area of the absorbent material. J. Acoust Soc Am 1963; 35(4):571-573.

\section{Appendix A. calculations of PTFs}

\section{A.1. PTFs for a parallelepiped cavity.}

Calculation of the PTFs of a parallelepiped backing cavity, shown is figure A.1, is illustrated. Consideringtime dependence $e^{j \omega t}$, the variational formulation of the problem can be expressed, for all test regular functions $\delta p$ on ${ }^{\Omega_{c}}$, as follows:

$$
\frac{1}{\rho_{0}} \int_{\Omega_{c}} \nabla p \nabla \delta p d V-\frac{\omega^{2}}{\rho_{0} c_{0}^{2}} \int_{\Omega_{c}} p \delta p d V=-j \omega \int_{\partial S_{i}} \delta p d S \text {. }
$$


Eigenfunctions $\phi_{q r s}$ and eigenfrequencies $\omega_{q r s}$ are available for a rigid-walled parallelepiped cavity of dimension $L_{x} \times L_{y} \times L_{z}$ :

$$
\begin{gathered}
L_{x} \times L_{y} \times L_{z}: \\
\omega_{q r s}=c_{0} \sqrt{\left(\frac{q \pi x}{L_{x}}\right)^{2}+\left(\frac{r \pi x}{L_{y}}\right)^{2}\left(\frac{s \pi x}{L_{z}}\right)^{2}}, \\
\phi_{q r s}(x, y, z)=\cos \left(\frac{q \pi x}{L_{x}}\right) \cos \left(\frac{r \pi y}{L_{y}}\right) \cos \left(\frac{s \pi z}{L_{z}}\right),
\end{gathered}
$$

The modal generalised mass $\mu_{\text {qrs }}$ is defined by:

$$
\mu_{q r s}=\frac{1}{\rho_{0} c_{0}{ }^{2}} \int_{\Omega_{c}} \phi_{q r s} \phi_{q r s} d V=\frac{1}{\rho_{0} \omega_{q r s}{ }^{2}} \int_{\Omega_{c}} \nabla \phi_{q r s} \nabla \phi_{q r s} d V \text {. }
$$

Using (A.3), one obtains:

$$
\mu_{q r s}=\frac{1}{8 \varepsilon_{q r s} \rho_{0} c_{0}^{2}}
$$

where $^{\varepsilon_{q r s}=\left(\frac{1}{2}\right)^{\Xi} \text { and }(q, r, s) \text { denotes the mode number }}$

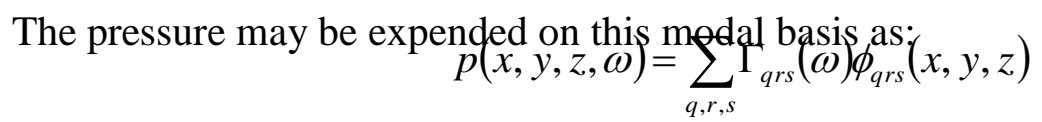

where $\Gamma_{q r s}$ are the modal amplitudes to be determined.

Introducing this modal expansion in the variational formulation and using $\delta p=\phi_{q r s}$ as test function, one obtains:

$$
\Gamma_{n m q}=-\frac{j \omega \int_{\partial S_{i}} \phi_{q r s} d S}{\mu_{q r s}\left(-\omega^{2}+\omega_{q r s}^{2}\right)} .
$$

Dissipation in the fluid medium can be taken into account by introducing a modal damping factor, $\eta_{c}$. This is done by adding an imaginary term in the denominator. Then, the PTF between excited patch $i$ and receiving patch $j, Z_{i j}$ is: 


$$
Z_{i j}=-\sum_{q, r, s} \frac{j \omega \int_{\partial S_{i}} \phi_{q r s} d S \int_{\partial S_{j}} \phi_{q r s} d S}{\mu_{q r s}\left(-\omega^{2}+j \omega \eta_{c} \omega_{q r s}+\omega_{q r s}^{2}\right)},
$$

and the PTF between the excited patch $i \underset{j \omega \phi_{q r s}}{\operatorname{and}}(M) \underset{\phi_{q r s}}{\phi_{S}} \operatorname{rejing}$ point $M$ inside the cavity is:

$$
Z_{i M}=-\sum_{q, r, s} \frac{\mu_{q S_{i}}\left(-\omega^{2}+j \omega \eta_{c} \omega_{q r s}+\omega_{q r s}^{2}\right)}{\mu^{2}} \text {. }
$$

\section{A.2. PTFs for a semi-infinite acoustic domain.}

One considers a semi-infinite acoustic domain where a unit normal velocity is prescribed on the patch $i$ and where the other patches are supposed to be rigid. The pressure at point $M$ inside or at the boundary of the acoustic domaing given by the Rayleigh integral equation:

$$
p_{M}=\frac{J \rho_{0} \omega}{2 \pi} \int_{\partial S_{i}} \frac{e^{y M \|}}{\| Q M} \text {. }
$$

$k_{0}=\frac{\omega}{c_{0}}$ is the acoustic wavenumber. Note that the integrand is singular (tends to infinity) as $\|Q M\|$ tends to zero. This problem which intervenes in the calculation of the PTF of patch $i, Z_{i i}$ must be overcome by the use of cylindrical coordinates by considering a circular patch having the same surface as the original one. In this case, the calculation of Cauchy's principal value of the integral gives:

$$
p_{M}=\rho_{0} c_{0}\left(1-e^{-j k_{0} a_{i}}\right)
$$

where ${ }^{a_{i}}$ is the radius of the equivalent circular patch:

$$
a_{i}=\sqrt{\frac{\partial S_{i}}{\pi}}
$$

The PTF of patch $i$ is then:

$$
Z_{i i}=\rho_{0} c_{0}\left(1-e^{-j k_{0} a_{i}}\right) \partial S_{i}
$$

For the PTF between patch $i$ and patch $j(i \neq j)$, the patch surfaces $\partial S_{i}$ and $\partial S_{j}$ are discretized in $K$ and $K^{\prime}$ elementary surfaces, respectively. As the distance $\|Q M\|$ is quasi- 
constant for Q belonging to an element surface, the integral of Eq. (A.10) can be approximated by:

$$
p_{M}=\frac{j \rho_{0} \omega \partial S_{i}}{2 \pi K} \sum_{k=1}^{K} \frac{e^{-j k_{0}\left\|Q_{k} M\right\|}}{\left\|Q_{k} M\right\|} .
$$

where $Q_{k}$ is the center point of the $k^{\text {th }}$ elementary surface.

Thus, the PTF between patch $i$ and patch $j_{\text {can }}$ be evaluated by:

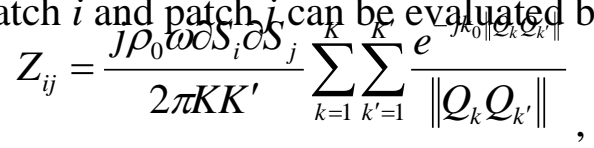

and the patch PTF between the patch $i$ and the receiving point $M^{\prime}$ inside the semi-infinite acoustic medium by:

$$
Z_{i M^{\prime}}=\frac{j \rho_{0} \omega \partial S_{i}}{2 \pi K} \sum_{k=1}^{K} \frac{e^{-j k_{0}\left\|Q_{k} M^{\prime}\right\|}}{\left\|Q_{k} M^{\prime}\right\|} .
$$




\section{Appendix B. Acoustic intensity calculation in a semi-infinite space}

The acoustic intensity at point $M^{\prime}$ in the direction $\xi(\xi \in\{x, y, z\})$ is defined by:

$$
I_{\xi}\left(M^{\prime}\right)=\frac{1}{2} \operatorname{Re}\left\{p_{M^{\prime}}^{1} u_{\xi, M^{\prime}}^{1}{ }^{*}\right\}
$$

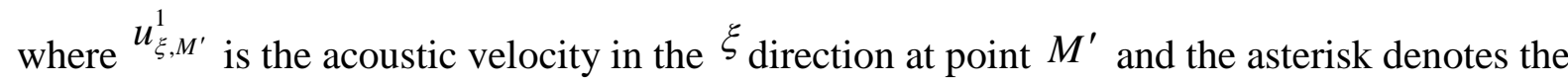
complex conjugate.

The pressure at point $M^{\prime}$ can be calculated from the patch velocities, ${ }^{1}{ }_{i}$ :

$$
p_{M^{\prime}}^{1}=\tilde{p}_{M^{\prime}}^{1}+\sum_{i=1}^{N} Z_{i M^{\prime}}^{1} u_{i}^{1}
$$

where the blocked pressure at point $M^{\prime}$ due to the incident wave is:

$$
\tilde{p}_{M^{\prime}}^{1}=2 p_{0} \cos \left[k_{0} \cos (\theta) z\right] e^{j\left[k_{0} \sin (\theta) x\right]} .
$$

In order to calculate the acoustic velocities at point $M^{\prime}$ with the same process, one should introduce a supplementary patch transfer function between patch $i$ and point $M^{\prime}, T_{\xi M^{\prime}}^{1}$ defined

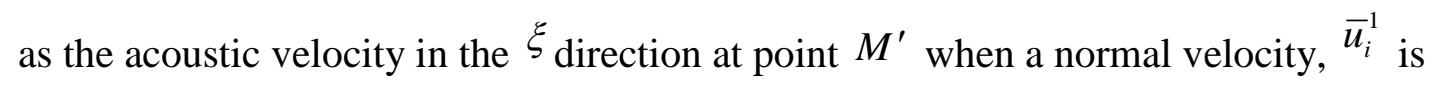
prescribed on patch $i$ (when other patches are supposed rigid):

$$
T_{\xi M^{\prime}}^{1}=\frac{u_{\xi, M^{\prime}}}{\bar{u}_{i}^{1}}
$$

where $u_{\xi, M^{\prime}}$ is the acoustic velocity in the $\xi_{\text {direction at point } M^{\prime}}$.

The particle velocity can be related to the pressure gradient using Euler's equation:

$$
u_{\xi, M^{\prime}}^{1}=\frac{j}{\rho_{0} \omega} \frac{\partial p_{M^{\prime}}}{\partial \xi} \text {. }
$$

The pressure gradient can be calculated from Eq. (A.14) when a unit normal velocity is prescribed on patch $i$ :

$$
\frac{\partial p_{M^{\prime}}}{\partial \xi}=\frac{j \rho_{0} \omega \partial S_{i}}{2 \pi K} \sum_{k=1}^{K} \frac{\overrightarrow{Q_{k} M^{\prime}} \cdot \vec{\xi}}{\left\|Q_{k} M^{\prime}\right\|}\left[-\frac{j k_{0} e^{-j k_{0}\left\|Q_{k} M^{\prime}\right\|}}{\left\|Q_{k} M^{\prime}\right\|}-\frac{e^{-j k_{0}\left\|Q_{k} M^{\prime}\right\|}}{\left\|Q_{k} M^{\prime}\right\|^{2}}\right]
$$

This leads to: 


$$
T_{\xi M^{\prime}}^{1}=\frac{\partial S_{i}}{2 \pi K} \sum_{k=1}^{K} \frac{\overrightarrow{Q_{k} M^{\prime}} \cdot \vec{\xi}}{\left\|Q_{k} M^{\prime}\right\|}\left[\frac{j k_{0} e^{-j k_{0}\left\|Q_{k} M^{\prime}\right\|}}{\left\|Q_{k} M^{\prime}\right\|}+\frac{e^{-j k_{0}\left\|Q_{k} M^{\prime}\right\|}}{\left\|Q_{k} M^{\prime}\right\|^{2}}\right]
$$

Using these PTFs and the patch velocities, ${ }^{1}{ }_{i}^{1}$ (obtained from Eq. (27)), the velocity at point $M^{\prime}$ in the $\xi_{\text {direction can be calculated from: }}$

$$
u_{\xi, M^{\prime}}^{1}=\tilde{u}_{\xi, M^{\prime}}^{1}+\sum_{i=1}^{N} T_{i M^{\prime}}^{1} u_{i}^{1}
$$

where the blocked velocity $\tilde{u}_{\xi, M^{\prime}}^{1}$ is determined by

$$
\tilde{u}_{\xi, M^{\prime}}^{1}=\frac{j}{\rho_{0} \omega} \frac{\partial}{\partial \xi}\left\{2 p_{0} \cos \left[k_{0} \cos (\theta) z\right] e^{j\left[k_{0} \sin (\theta) x\right]}\right\}
$$

Finally, the acoustic intensity at point $M^{\prime}$ in the direction $\xi$ can be obtained by introducing Eq. (B.2) and (B.8) in the relation (B.1). 
TABLE

\begin{tabular}{|l|l|l|l|l|l|l|l|l|l|l|l|l|}
\hline$f_{q, r, s}(\mathrm{~Hz})$ & 0 & 340 & 481 & 567 & 660 & 680 & 743 & 760 & 885 & 948 & 961 & 1020 \\
\hline$(q, r, s)$ & $0,0,0$ & $0,1,0$ & $1,1,0$ & $0,0,1$ & $0,1,1$ & $0,2,0$ & $1,1,1$ & $1,2,0$ & $0,2,1$ & $1,2,1$ & $2,2,0$ & $0,3,0$ \\
& & $1,0,0$ & & & $1,0,1$ & $2,0,0$ & & $2,1,0$ & $2,0,1$ & $2,1,1$ & & $3,0,0$ \\
\hline
\end{tabular}

\begin{tabular}{|l|l|l|l|l|l|l|l|l|l|l|l|l|}
\hline$F_{q, r, s}(H z)$ & 1075 & 1116 & 1133 & 1167 & 1183 & 1215 & 1226 & 1231 & 1321 & 1350 & 1360 & 1364 \\
\hline$(q, r, s)$ & $1,3,0$ & $2,2,1$ & $0,0,2$ & $0,3,1$ & $0,1,2$ & $1,3,1$ & $2,3,0$ & $1,1,2$ & $0,2,2$ & $2,3,1$ & $0,4,0$ & $1,2,2$ \\
& $3,1,0$ & & & $3,0,1$ & $1,0,2$ & $3,1,1$ & $3,2,0$ & & $2,0,2$ & $3,2,1$ & $4,0,0$ & $2,1,2$ \\
\hline
\end{tabular}

Table 1. Natural frequencies of the rectangular cavity with rigid boundaries. 


\section{FIGURES}

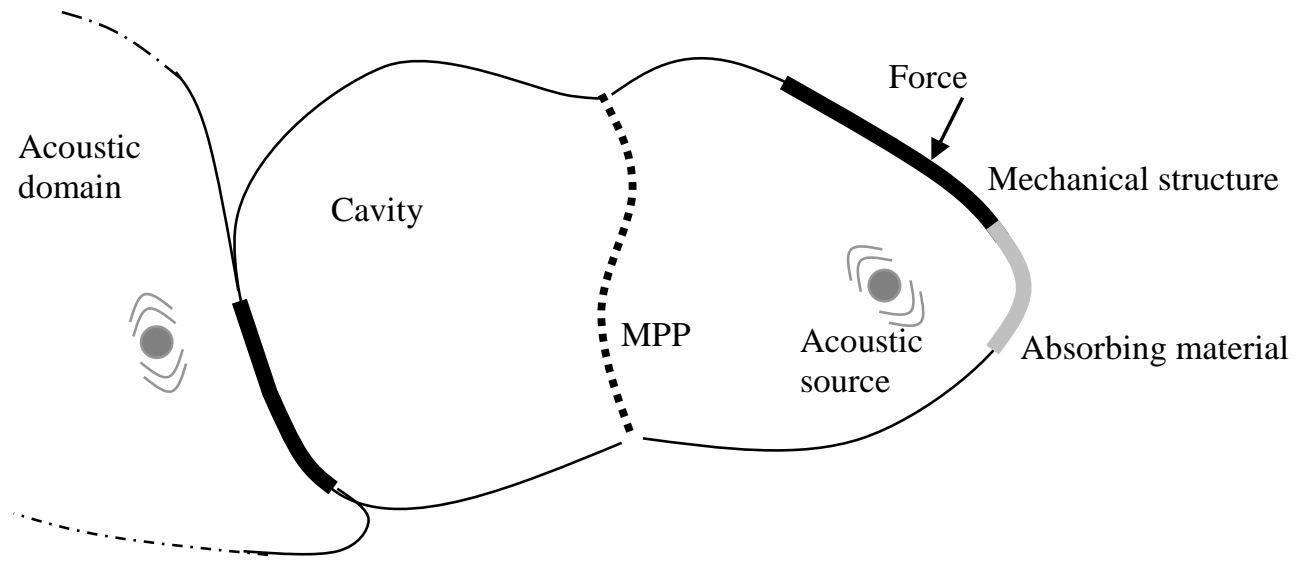

(a)
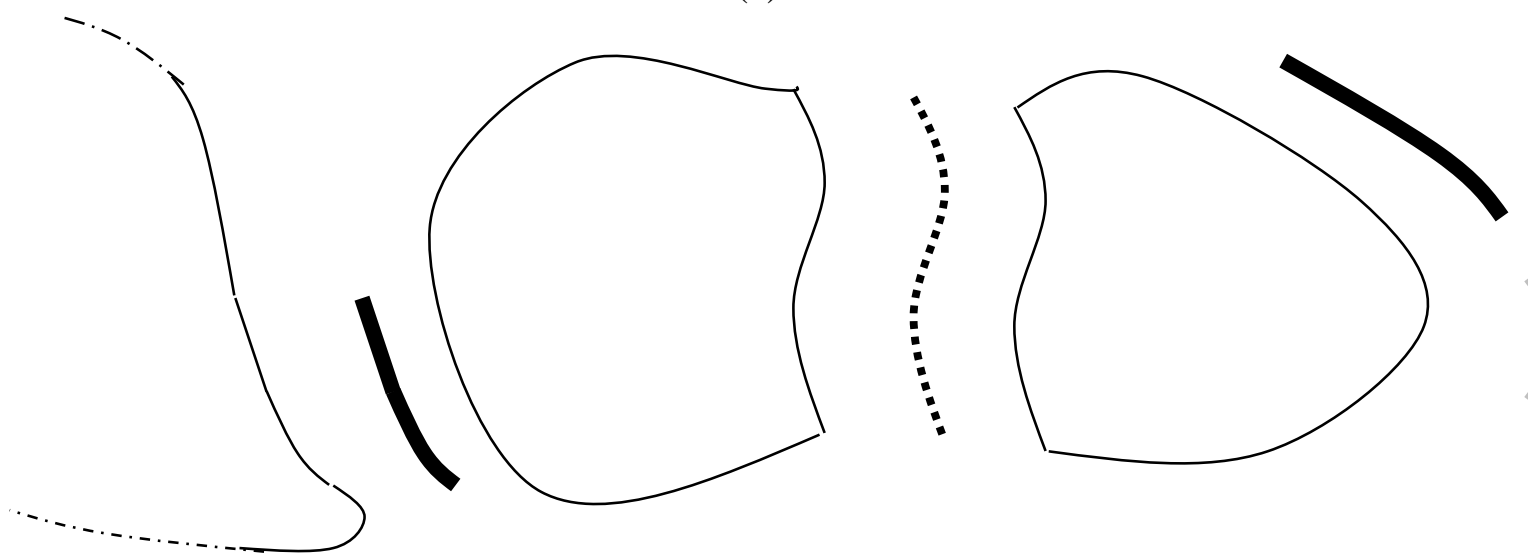

(b)

Figure 1. (a), MPP in a practical vibro-acoustic environment. (b), PTF substructuring. 


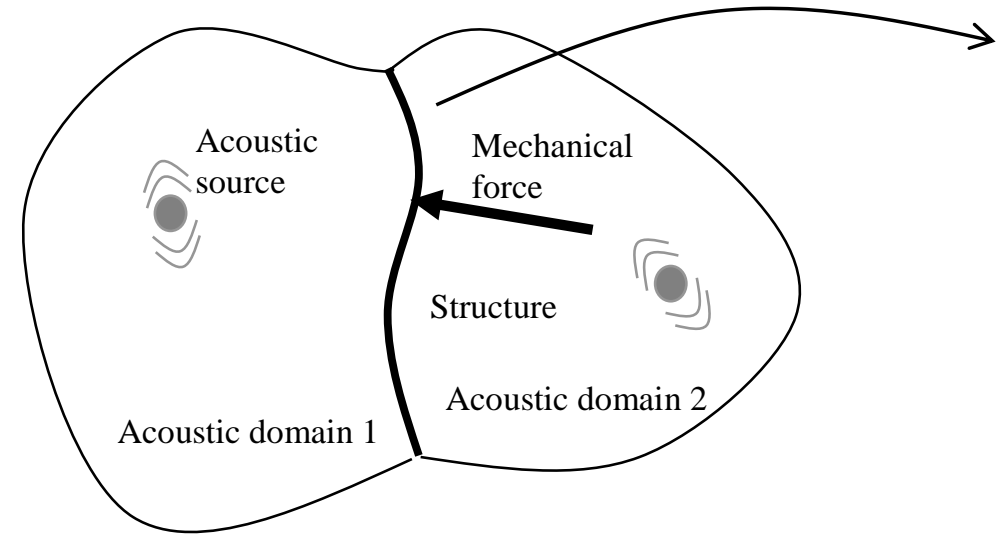

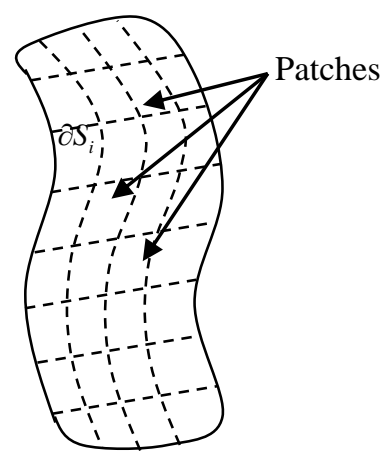

Coupling surface

Figure 2. (a), Example of a vibro-acoustic problem; (b), Coupling surface divided into $N$ patches.

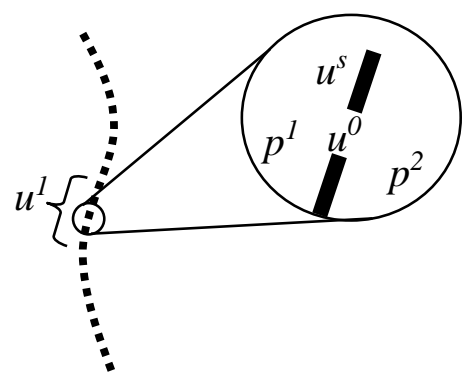

Figure 3. Description of pressure and velocity variables for the MPP. 


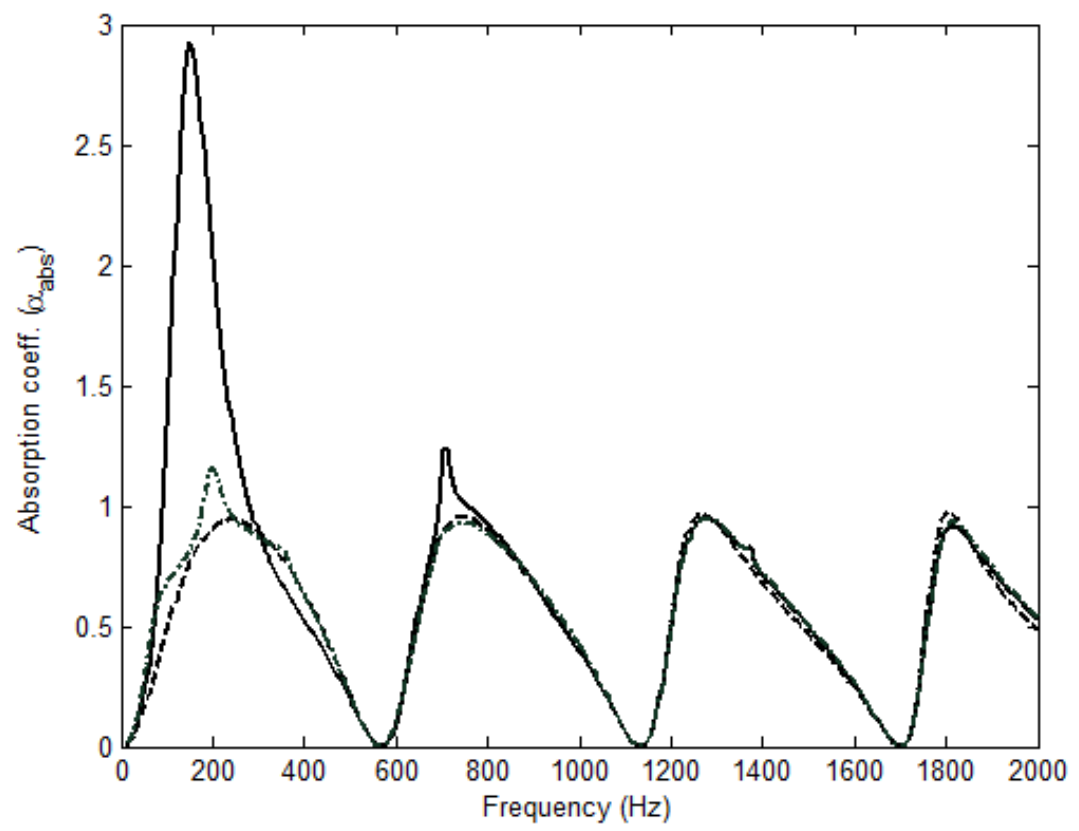

Figure 4. Absorption coefficient versus frequency. Solid line: PTF result for a $0.5 \mathrm{~m} \times 0.5 \mathrm{~m}$ MPP; Dash-dotted: PTF result for a 2m x2m MPP; Dash: equivalent circuit result. 

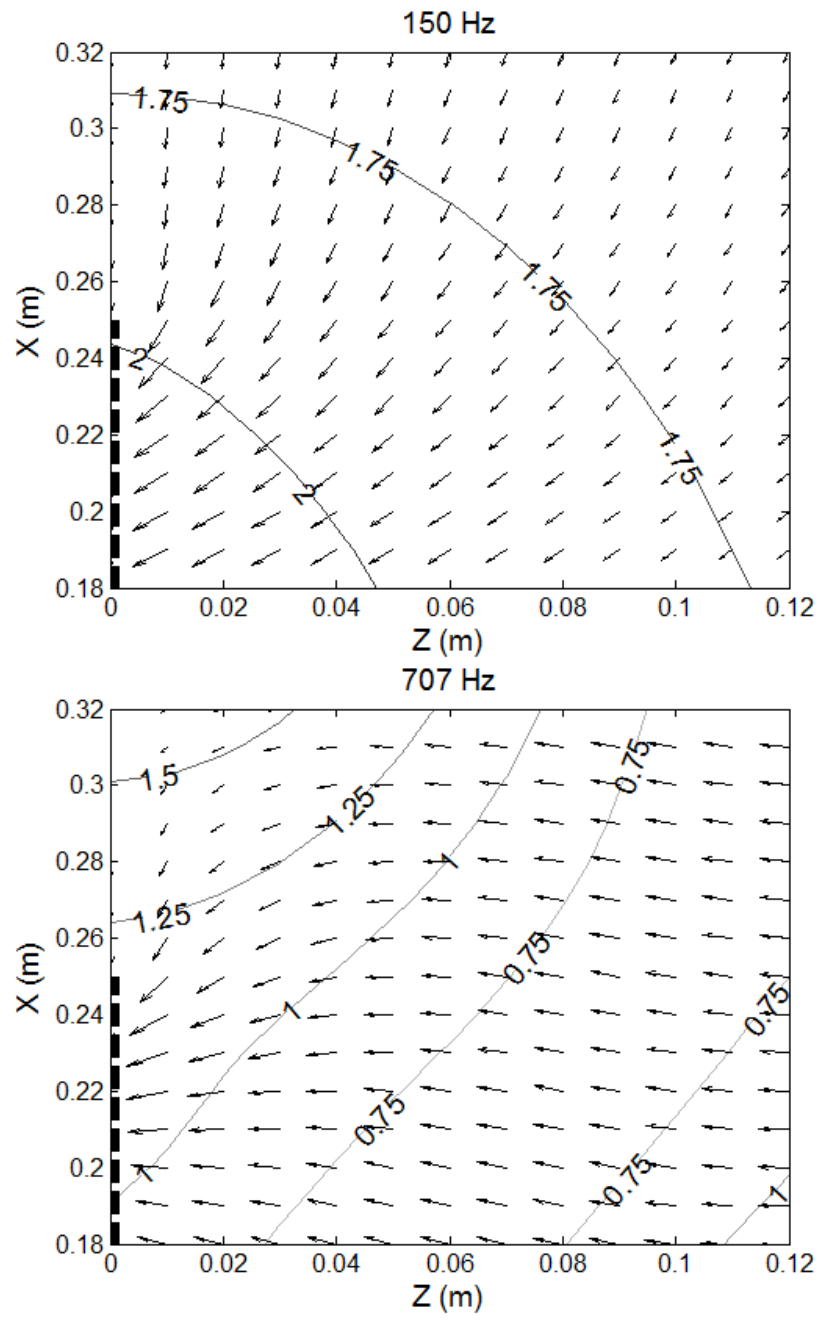

(b)

$1267 \mathrm{~Hz}$

(c)

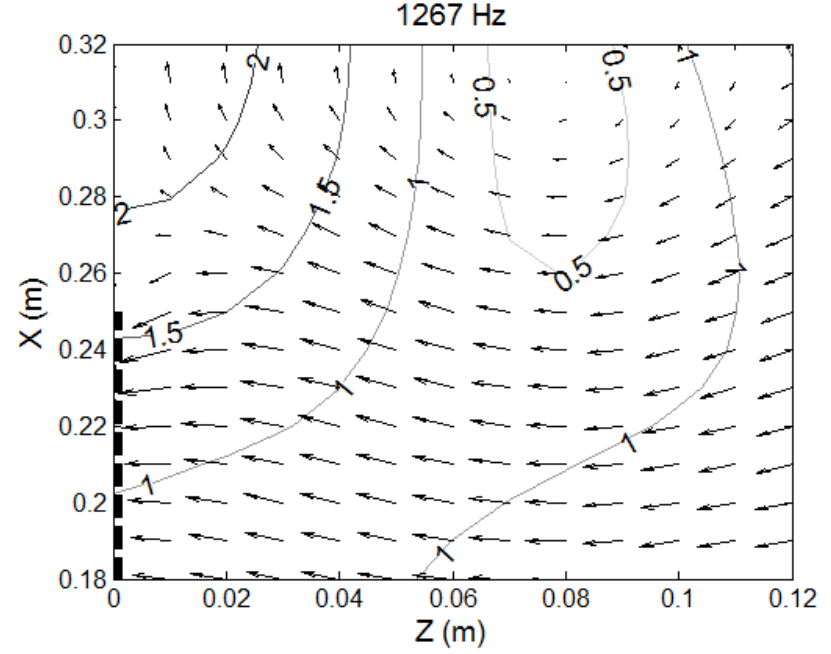

Figure 5. Acoustic intensity (arrow) and acoustic pressure (contour line) in a plane at $\mathrm{Y}=0.25 \mathrm{~m}$ in the front of the MPP. Normal incidence of the plane wave. MPP position symbolised by a dash line. (a), $150 \mathrm{~Hz}$; (b), $707 \mathrm{~Hz}$; (c), $1267 \mathrm{~Hz}$. 

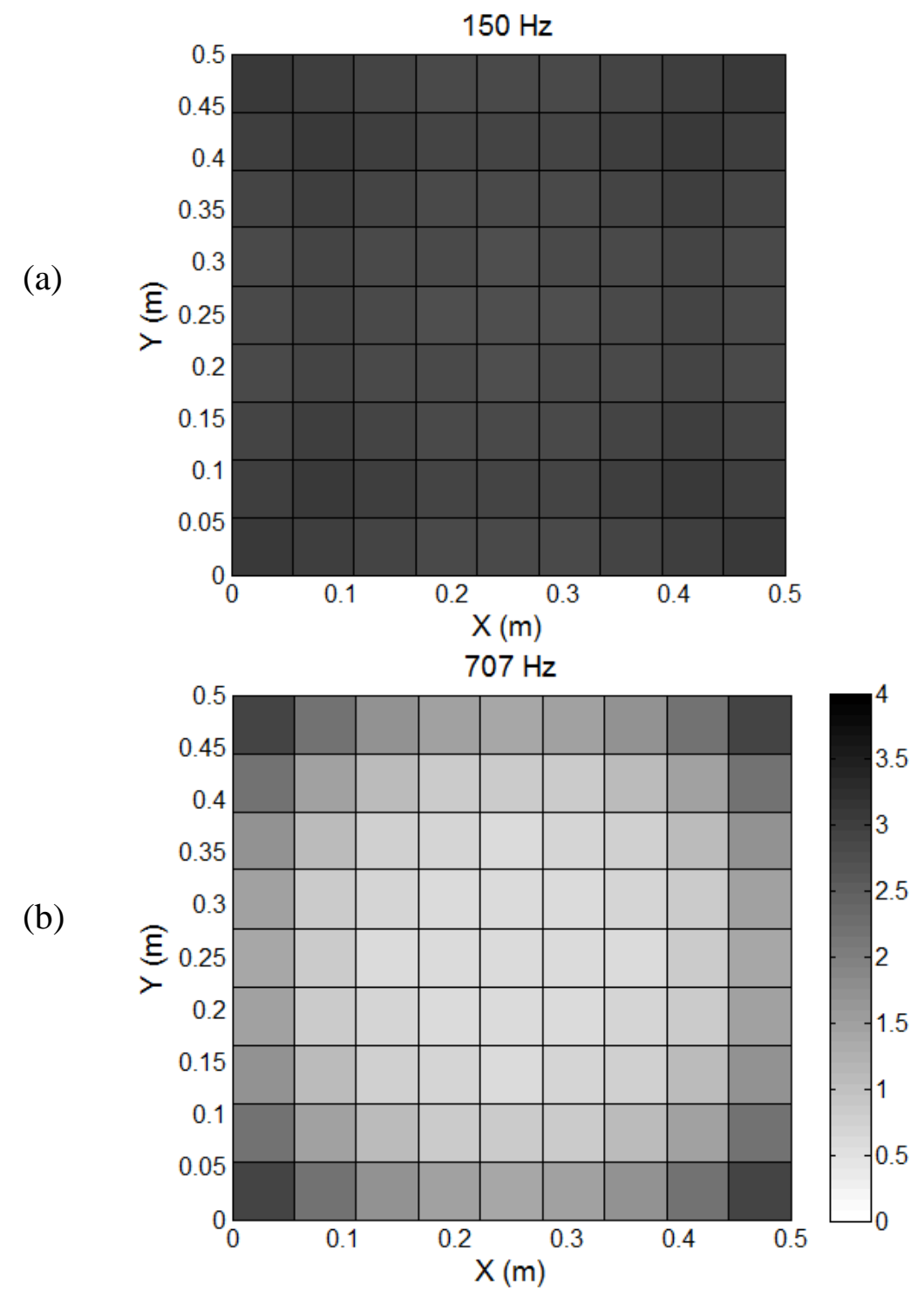

(b)

$1267 \mathrm{~Hz}$

(c)

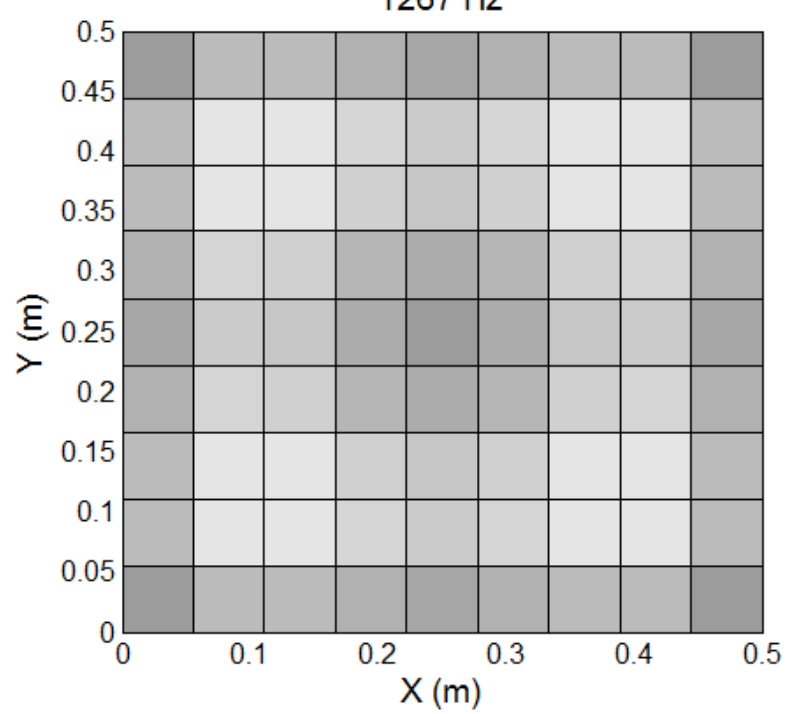

Figure 6. Local patch absorbing coefficients. (a), $150 \mathrm{~Hz}$; (b), $707 \mathrm{~Hz}$; (c), $1267 \mathrm{~Hz}$. 
$150 \mathrm{~Hz}$

(a)

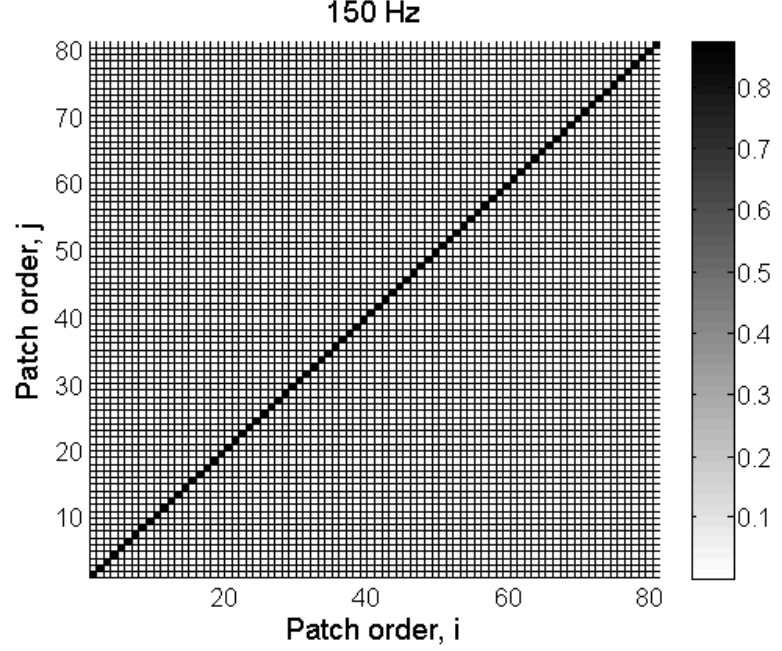

(b)

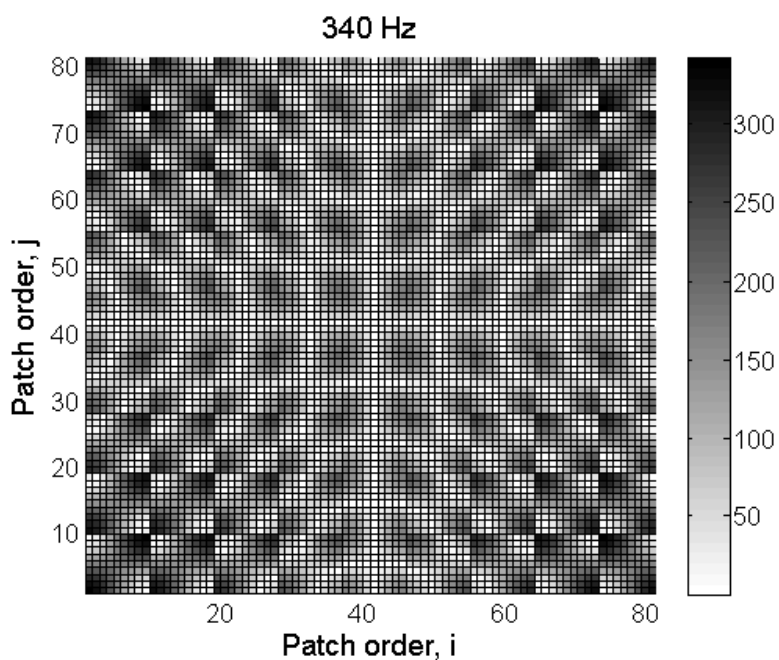

$567 \mathrm{~Hz}$

(c)

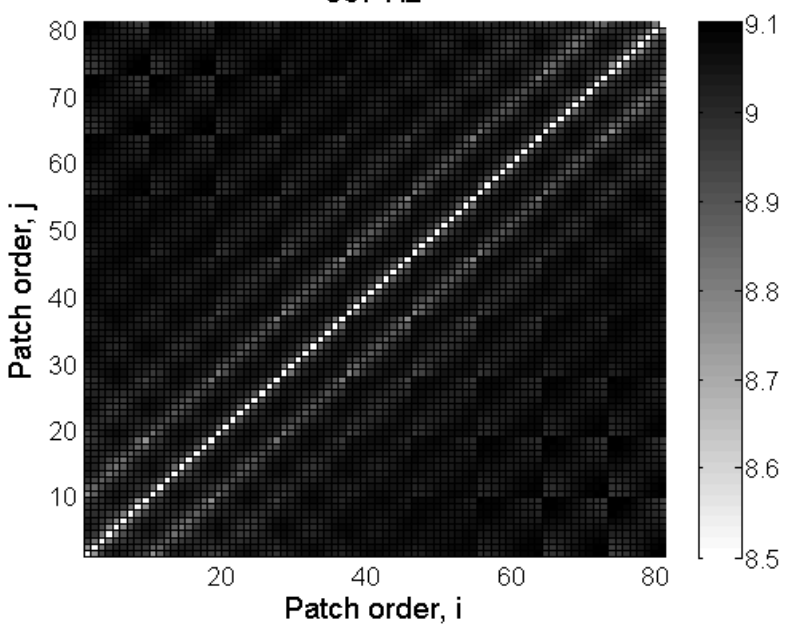

Figure 7. Magnitude of the Equivalent Patch Transfer Functions (N/(m/s)) 

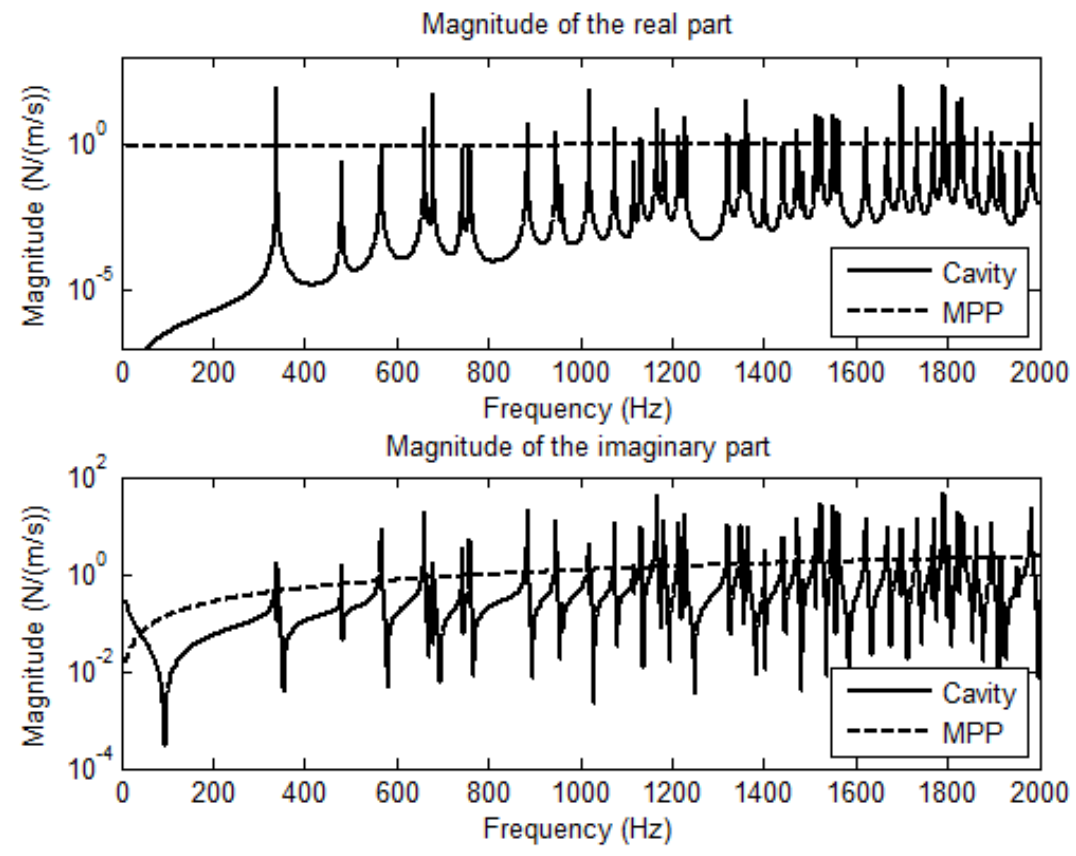

Figure 8. Comparison of the term $\frac{\partial S}{\Psi}$ of MPP with the PTF of the cavity. 


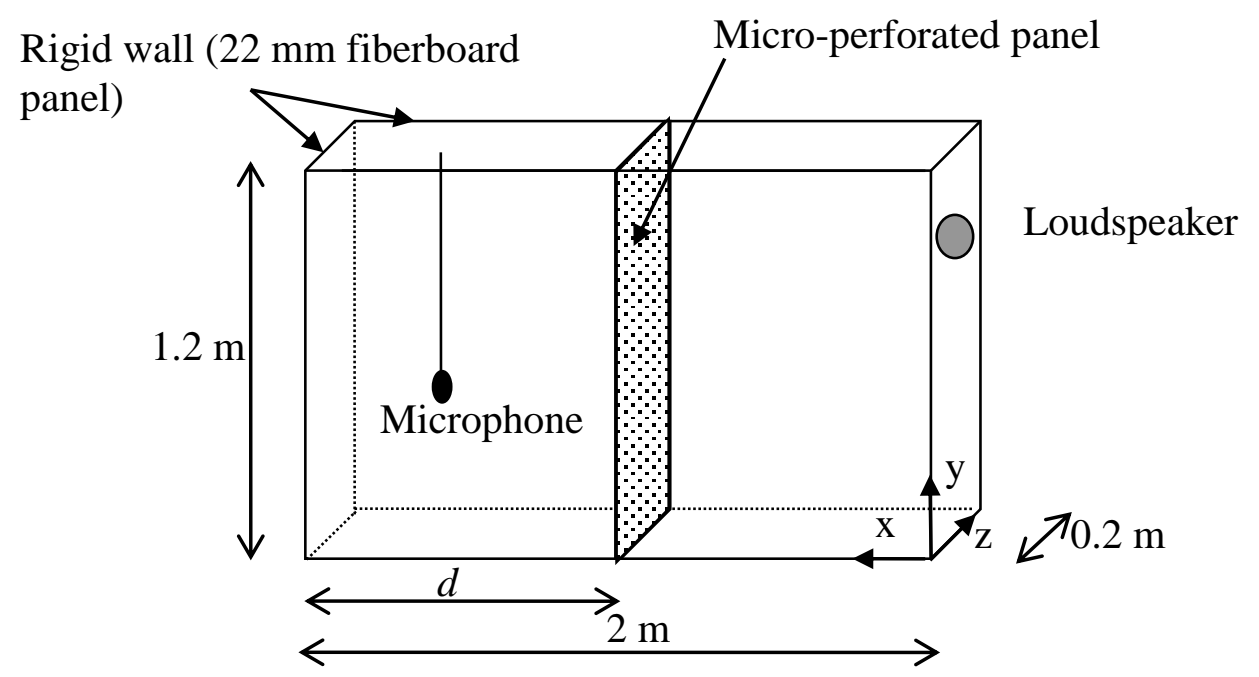

Figure 9. Rectangular cavity used in the experiments by Fenech et al. [10].
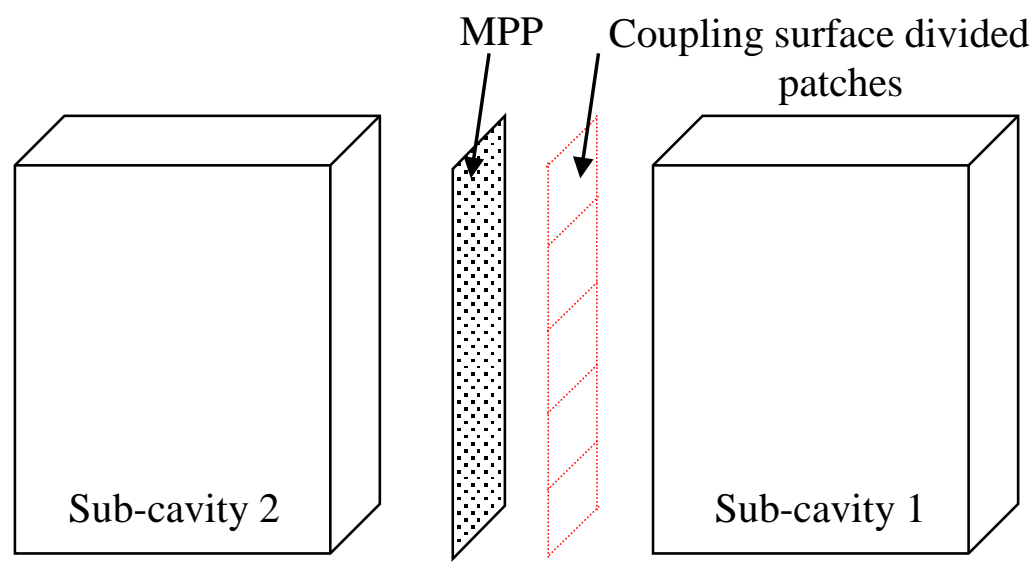

Figure 10. PTF substructuring and patch definition. 


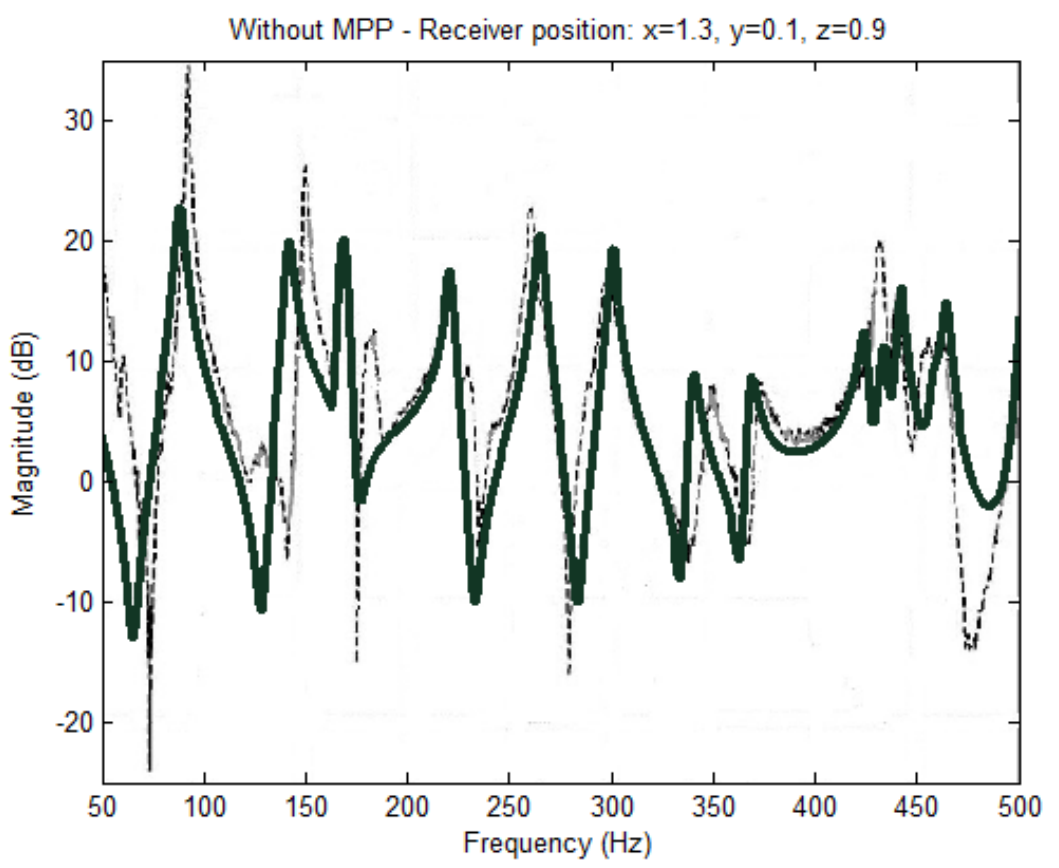

(a)

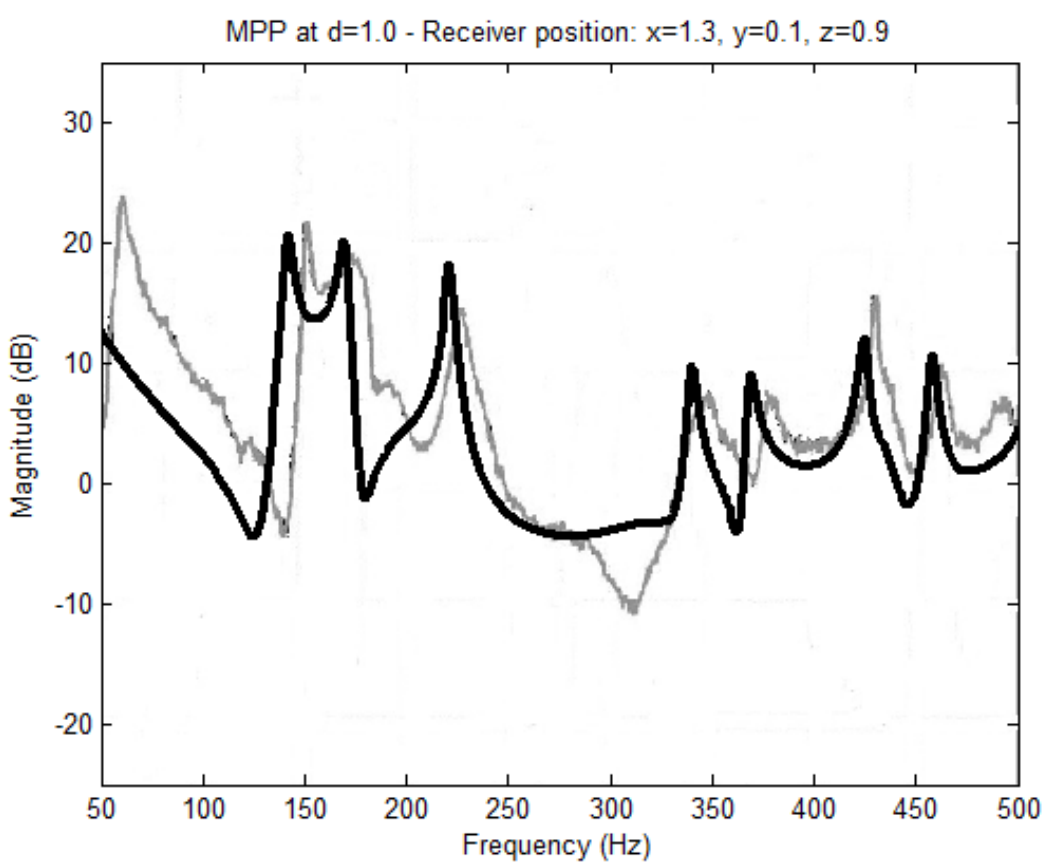

(b)

Figure 11. Comparison of sound pressure calculated by PTF (Solid line) with the measurement by Fenech et al. (Grey line) for two cases: (a) empty cavity; (b) with MPP at $\mathrm{d}=1.0 \mathrm{~m}$. 


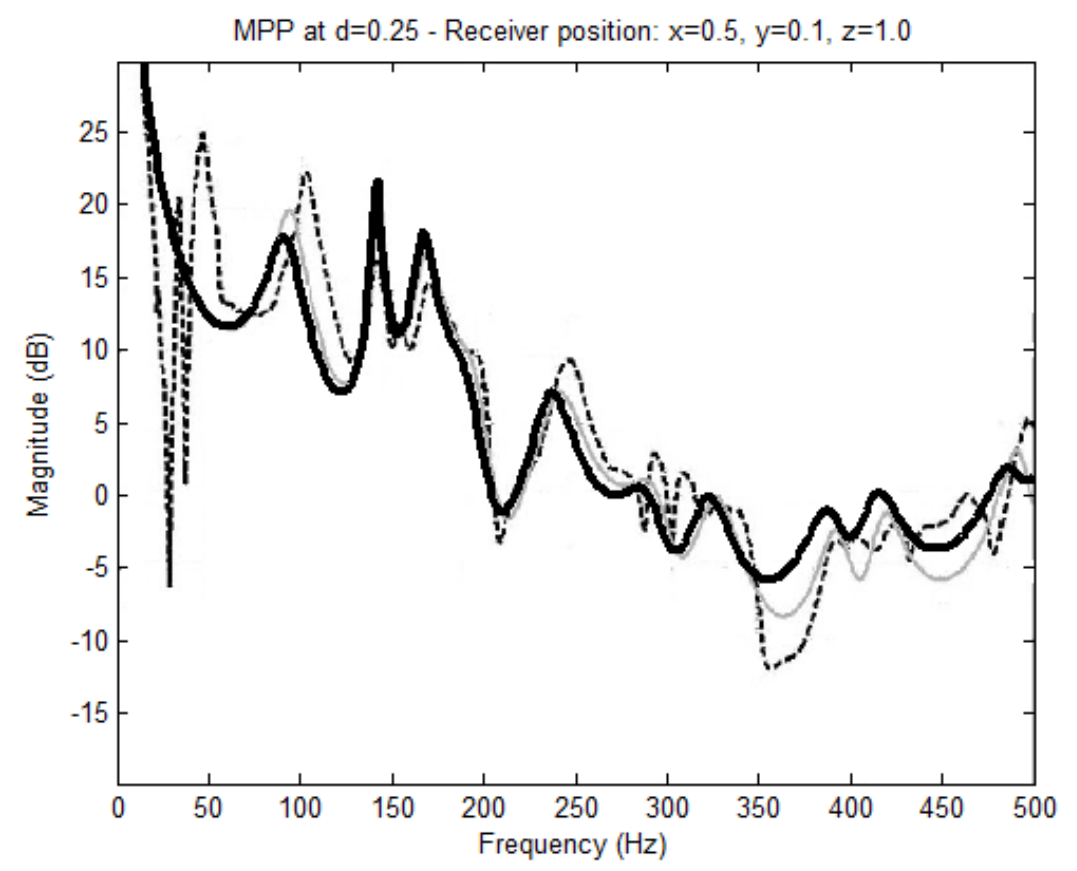

Figure 12. Comparison of PTF results with the pressures measured and calculated by Fenech et al. for a MPP at $\mathrm{d}=0.25 \mathrm{~m}$. Solid line: PTF results; Dash line, Fenech' measurement; Grey line: Fenech' prediction;

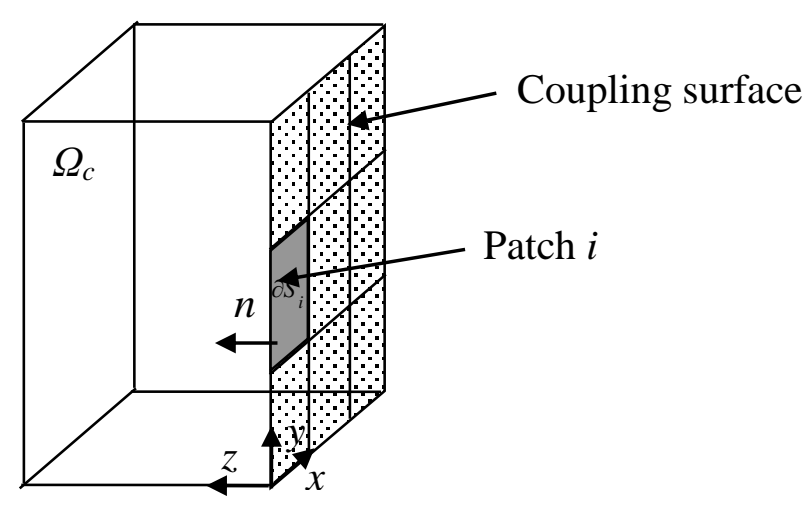

Figure A.1. A rigid-walled parallelepiped cavity with a unit normal velocity prescribed on the patch surface $\partial S_{i}$. 